D. Popescu

Nagoya Math. J.

Vol. 104 (1986), 85-115

\title{
GENERAL NÉRON DESINGULARIZATION AND APPROXIMATION
}

\author{
DORIN POPESCU
}

\section{§1. Introduction}

Let $A$ be a noetherian ring (all the rings are supposed here to be commutative with identity), $\mathfrak{a} \subset A$ a proper ideal and $\hat{A}$ the completion of $A$ in the a-adic topology. We consider the following conditions

(WAP) Every finite system of polynomial equations over $A$ has a solution in $A$ iff it has one in $\hat{A}$.

(AP) Every finite system of polynomial equations over $A$ has its set of solutions in $A$ dense with respect to the $a$-adic topology in the set of its solutions in $\hat{A}$, i.e. for every solution $\hat{y}$ from $\hat{A}$ and every positive integer $c \in N$ there exists a solution $y_{c}$ in $A$ such that $y_{c} \equiv \hat{y} \bmod \mathfrak{a}^{c} \hat{A}$.

(SAP) For every finite system of polynomial equations $f$ over $A$ there exists a function $\nu: N \rightarrow N$ with the following property:

"For every positive integer $c$ and for every system of elements $\tilde{y}$ from $A$ which form a solution of $f$ modulo $\mathfrak{a}^{2(c)}$ there exists a solution $y_{c}$ of $f$ in $A$ such that $\tilde{y} \equiv y_{c} \bmod \mathfrak{a}^{c} "$.

It is easy to note that (SAP) is stronger than (AP) and (WAP) is equivalent with (AP) (see (3.3.1)). We call $(A, \mathfrak{a})$ an $(A P)$-couple (resp. $(S A P)$-couple) if it satisfies (AP) (resp. (SAP)). A local ring $(A, \mathfrak{m})$ is called a ring with the property of Artin approximation or an AP-ring if the couple $(A, \mathfrak{m})$ is an AP-couple.

Examples of (non complete) AP-rings are mainly given by the following Theorems:

(1.1) Theorem (M. Artin $\left.\left[\mathrm{A}_{1}\right]\right)$. The convergent power series rings in $X=\left(X_{1}, \cdots, X_{n}\right)$ over a non-trivial valued field of characteristic zero are AP-rings.

(1.2) Theorem (M. Artin $\left.\left[\mathrm{A}_{2}\right]\right)$. The henselization of a local ring which

Received February 25, 1985. 
is essentially of finite type over a field or an excellent Dedekind ring is an AP-ring.

Theorem (1.1) has also some forms in characteristic $p>0$ (see $\left[\mathrm{An}_{3}\right]$, [J]). Recently K. P. Schemmel [S] showed that a convergent power series ring $R$ in some variables over a valued field is an AP-ring iff it is universally japanese (in this case, iff it is excellent).

Theorem (1.2) has some extensions in [KMPPR], $\left[\mathrm{P}_{2}\right],[\mathrm{B}],[\mathrm{CP}],\left[\mathrm{P}_{3}\right]$. The main purpose of our paper is to prove the following Theorem (see Corollary (3.3)).

(1.3) Theorem. A noetherian henselian couple $(A, \mathfrak{a})$ is an AP-couple if the completion morphism $A \rightarrow \hat{A}$ in the a-adic topology is regular. In particular excellent henselian local rings are AP-rings.

The above Theorem gives a positive answer to $\left[A_{3}\right]$ Conjecture (1.3). As a consequence we get that the completion of an excellent henselian factorial ring is factorial too (see (3.4)). Also using Theorem (1.3) we solve completely the question of so called approximation on nested subrings which is the subject of [KMPPR], Ch. III, $\left[\mathrm{PP}_{2}\right]$ and [BDL], i.e. we get in particular the following.

(1.4) Theorem. Let $k$ be a field, $k\langle X\rangle$ the algebraic power series ring in $X=\left(X_{1}, \cdots, X_{r}\right)$ over $k, f$ a finite system of polynomial equations over $k\langle X\rangle$ and $\hat{y}=\left(\hat{y}_{1}, \cdots, \hat{y}_{n}\right) \in k \llbracket X \rrbracket^{n}$ a formal solution of $f$ such that $\hat{y}_{i} \in k \llbracket X_{1}$, $\cdots, X_{s_{i}} \rrbracket, 1 \leqslant i \leqslant n$ for some positive integers $s_{i} \leqslant r$. Then there exists a solution $y=\left(y_{1}, \cdots, y_{n}\right)$ of $f$ in $k\langle X\rangle$ such that $y_{i} \in k\left\langle X_{1}, \cdots, X_{s_{i}}\right\rangle, 1 \leqslant i \leqslant n$.

The above Theorem-a particular case of Theorem (3.7)-is very useful in deformation theory, but our paper contains no such applications. Some ideas about them can be found in [KMPPR] $\mathrm{Ch}$. IV.

A noetherian couple $(A, \mathfrak{a})$ is called a couple with the property of strong approximation, or a (SAP)-couple if it satisfies (SAP). Clearly a (SAP)-couple is an AP-couple. When $(A, \mathfrak{a})$ is local it is true also the converse implication as shows the following.

(1.5) Theorem (Pfister-Popescu). (SAP) holds for noetherian complete local rings.

This is a particular form of $\left[\mathrm{PP}_{1}\right]$ Theorem (2.5); its proof was given in an improved form in [KMPPR] Ch. II Theorem (1.4), another proof in- 
volving ultrapowers was given in [DL] and using also the ultrapowers we arranged in $\left[\mathrm{P}_{1}\right]$ some other versions of Theorem (1.4) (ibid). In our Section 4 we get very short proofs to Theorem (1.5) and to an Elkik result [E] (see Corollary (4.5) and Corollary (4.7)..

It remains still open the following questions (see Remark (4.6)).

(1.6) Question. Is a noetherian complete couple a (SAP)-couple?

(1.7) Question. Is an AP-ring excellent?

The last question is true for rings of dimensions $\leq 2$. In general $\mathrm{C}$. Rotthaus proved that an AP-ring $A$ is excellent if $A[X]$ is also an APring (see [Ro]).

The proof of Theorem (1.3) (in fact of Theorem (3.2)) use an extension of Néron desingularization which says roughly speaking the following:

(1.8) Theorem. A morphism of noetherian rings is regular iff it is a filtered inductive limit of finite type smooth morphisms.

This theorem is a variant of Theorem (2.5) and it was already proved in $\left[\mathrm{P}_{4}\right]$ under some separability conditions. Its proof is given in Section 2, Sections 6-10, following the ideas from $\left[\mathrm{P}_{4}\right]$. Our Lemma (7.4) was inspirated from [AD], Lemma (4.12) and [Gr]. As applications of the above Theorem we get a result of André [ $\left.\mathrm{An}_{1}\right]$ (see our Corollary (2.6)) and a partial positive answer to a question from EGA, Part 1, (19.7.3) (see our Theorem (5.5)).

Finally we want to mention that the results in $\left[\mathrm{P}_{4}\right]$ and in the present paper appeared in preprint form as: INCREST Preprint no. 44 and 49 (1983).

We would like to thank to $H$. Kurke and G. Pfister for pointing to us in 1977 the idea of the present Lemma (3.6). Also we want to thank to V. Nica and N. Radu for useful conversations on Theorems (4.4) resp. (5.5).

\section{§2. General Néron desingularization}

(2.1) Let $B \cong A[Y] /(f), Y=\left(Y_{1}, \cdots, Y_{n}\right), f=\left(f_{1}, \cdots, f_{m}\right)$ be a finite presentation algebra over a ring $A$, the isomorphism being given by $Y \rightarrow$ $y \in B^{n}$. Given a system $g=\left(g_{1}, \cdots, g_{r}\right), r \leqslant n$ of polynomials from $(f)$, we consider the ideal $\Delta_{g}$ generated in $A[Y]$ by all $r \times r$-minors of the Jacobian matrix $(\partial g / \partial Y)$ associated to $g$. Denote $H_{f}:=\sqrt{ }(f)+\sum_{g} \Delta_{g}((g):(f))$, the sum being taken over all systems $g$ of $r$-polynomials from $(f), r, 1 \leqslant r \leqslant n$ 
being variable. Then the ideal $H_{B / A}=H_{f}(y) \subset B$ does not depend of the chosen presentation for $B$ over $A$ and $V\left(H_{B / A}\right)$ is exactly the nonsmooth locus of $B$ over $A$. Thus $B$ is smooth over $A$ iff $H_{B / A}=B$. The above presentation is a standard smooth presentation if there exists a system $g$ of polynomials from $(f)$ such that $B=\left(\Delta_{g}((g):(f))\right)(y)$, i.e. there exists a polynomial $P \in \Delta_{g}((g):(f))$ such that $1 \in P+(f)$. In other words the above presentation is standard smooth if (and only if) there exist a system of polynomials $g$ in $(f)$ and a polynomial $P \in \Delta_{g}$ such that $B \cong(A[Y] /(g))_{P}$, the isomorphism being given by $Y \rightarrow y . \quad B$ is a standard smooth $A$-algebra if it has a standard smooth presentation and a ring morphism $h: A \rightarrow C$ is standard smooth if $C$ is a standard smooth $A$-algebra, the algebra structure on $C$ being given by $h$. As above $B$ is standard smooth $A$-algebra if it has a presentation of the form $B \cong(A[Y] / g)_{P}$, where $P \in \Delta_{g}$. Let $f$ : $B \rightarrow A^{\prime}$ be a morphism of $A$-algebra and $u: A \rightarrow A^{\prime}$ the algebra structure morphism. The couple $(B, f)$ has a desingularization with respect to $u$ if there exist a standard smooth $A$-algebra $B^{\prime}$ and two $A$-morphisms $v: B \rightarrow B^{\prime}$. $w: B^{\prime} \rightarrow A^{\prime}$ such that $w v=f$.

The following Lemma is just a variant of [AD] Lemma (5.2).

(2.2) Lemma. Let $u: A \rightarrow A^{\prime}$ be a ring morphism. Then the following conditions are equivalent:

i) $u$ is a filtered inductive limit of standard smooth A-algebras,

ii) for every finite presentation A-algebra $B$ and every A-morphism $f: B \rightarrow A^{\prime}$, the couple $(B, f)$ has a desingularization with respect to $u$.

To show that the separability conditions under which we proved Theorem (5.2) and Corollary (5.4) in $\left[\mathrm{P}_{4}\right]$ are superfluous, it is necessary to extend $\left[\mathrm{P}_{4}\right]$. Desingularization Lemma, i.e. to prove the following.

(2.3) Desingularization Theorem. Let $u: A \rightarrow A^{\prime}$ be a morphism of noetherian rings, $B$ a finite type A-algebra, $f: B \rightarrow A^{\prime}$ an $A$-morphism such

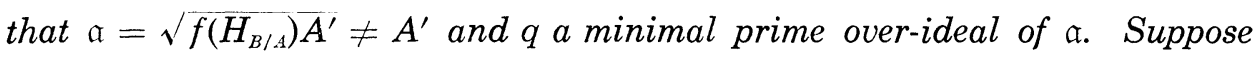
that

i) $A_{q}^{\prime}$ is a formally smooth $A_{u-1}$-algebra,

ii) for every minimal prime over-ideal $q^{\prime}$ of a the map $A \rightarrow A_{q^{\prime}}^{\prime}$, induced by $u$ is flat and $A_{q^{\prime}}^{\prime}\left(u^{-1} q^{\prime}\right) A_{q^{\prime}}^{\prime}$, is a regular ring,

iii) $A^{\prime} / q$ is not finite.

Then there exist a finite type A-algebra $B^{\prime}$ and two A-morphisms $v$ : $B \rightarrow B^{\prime}, w: B^{\prime} \rightarrow A^{\prime}$ such that 
(*) $\quad w v=f$

$(* *) \quad \mathfrak{a} \subset \sqrt{w\left(H_{B^{\prime} / A}\right) A^{\prime}} \not \subset q$.

The proof is difficult and will be given in Sections 6-10.

(2.4) Corollary. Let $u: A \rightarrow A^{\prime}$ be a morphism of noetherian rings and $F \subset \operatorname{Spec} A^{\prime}$ a closed set such that $u$ is regular on $F$, i.e. $A_{q}^{\prime}$ is a formally smooth A-algebra for every $q \in F$. Then for every finite type A-algebra $B$ and every morphism of A-algebras $f: B \rightarrow A^{\prime}$ such that $V\left(a_{f}\right) \subset F$, $a_{f}:=\sqrt{f\left(H_{B / A}\right) A^{\prime}}$ the couple $(B, f)$ has a desingularization with respect to $u$.

This Corollary extends $\left[\mathrm{P}_{4}\right]$ Theorem (5.2); its proof goes similarly to the quoted Theorem using Desingularization Theorem instead $\left[\mathrm{P}_{4}\right]$ Desingularization Lemma.

(2.5) Theorem. Let $u: A \rightarrow A^{\prime}$ be a morphism of noetherian rings. Then the following conditions are equivalent:

i) $u$ is regular,

ii) for every finite type A-algebra $B$ and every $A$-morphism $f: B \rightarrow A^{\prime}$ the couple $(B, f)$ has a desingularization with respect to $u$,

iii) $A^{\prime}$ is a filtered inductive limit of standard smooth A-algebras,

iv) $A^{\prime}$ is a filtered inductive limit of finite type smooth A-algebras.

Proof. i) $\Rightarrow$ ii) is a consequence of Corollary (2.4) ii) $\Leftrightarrow$ iii) is a consequence of Lemma (2.2) and iii) $\Rightarrow$ iv) is trivial.

iv) $\Rightarrow$ i) It is easy to see that a noetherian local ring which is a filtered inductive limit of regular local rings, is itself a regular local ring. Thus a filtered inductive limit of regular morphisms is regular if its cosource is noetherian.

Q.E.D.

(2.6) Conollary (André $\left.\left[\mathrm{An}_{1}\right]\right)$. Let $u: A \rightarrow A^{\prime}$ be a regular morphism of noetherian rings. Then $H_{1}\left(A, A^{\prime},-\right)=0\left(H_{1}\left(A, A^{\prime},-\right)\right.$ denotes the first homology functor).

Proof (after [CP]). If $B$ is a finite type smooth $A$-algebra then $H_{1}(A$, $B,-)=0$. By Theorem (2.5) $A^{\prime}$ is a filtered inductive limit of finite type smooth $A$-algebras. Then $H_{1}\left(A, \underset{i}{\lim } B_{i},-\right)=\underset{i}{\lim } H_{1}\left(A, B_{i},-\right)=0$. Q.E.D.

(2.6.1) Note. The above Corollary has in $\left[\mathrm{An}_{1}\right]$ a very difficult proof involving the homology functors of higher dimensions. N. Radu asks for a proof of this result involving only the homology functors of dimension $\leq 1$. Our proof can be an answer, but not too easy. The converse is 
also true via Jacobian criterion of formal smoothness.

(2.7) Corollary. Let $k$ be a field and $R$ a noetherian local $k$-algebra whose residue field is a separable field extension of $k$. Then the following conditions are equivalent:

i) $R$ is regular,

ii) $R$ is a filtered inductive limit of regular local rings essentially of finite type over $k$,

iii) $R$ is a filtered inductive limit of finite type smooth $k$-algebras.

The proof is a consequence of Theorem (2.5) via [M] (28.L) and [EGA], Part 1, (22.5.8).

(2.7.1) Note. When $R$ is a discrete valuation ring and char $k=0$, Zariski Uniformization Theorem says that $R$ is a filtered inductive limit of its finite type smooth sub-k-algebras. If $R$ is a regular ring of dimension $>1$, then we do not think that such a result is true. However we have no counterexamples.

\section{$\S 3$. Rings with the property of Artin approximation and AP-couples}

One reason to deal with standard smooth algebras instead of general finite type smooth algebras is given by the following "global" form of the Implicit Function Theorem.

(3.1) Lemma. Let $(A, \mathfrak{a})$ be a henselian couple and $B$ a standard smooth $A$-algebra. Then every A-morphism $B \rightarrow A /$ a can be lifted to an A-morphism $B \rightarrow A$.

Proof. Let $B \cong A[Y] /(f), Y=\left(Y_{1}, \cdots, Y_{n}\right), f=\left(f_{1}, \cdots, f_{s}\right)$ be a standard smooth presentation of $B$ over $A$ and $\bar{\sigma}: B \rightarrow A / \mathfrak{a}$ an $A$-morphism. Suppose that the map $A[Y] /(f) \rightarrow A / \mathfrak{a}$ induced by $\bar{\sigma}$ is given by $Y \rightarrow \tilde{y} \in A^{n}$. Since the above presentation is standard smooth there exists a system of polynomials $g=\left(g_{1}, \cdots, g_{r}\right)$ such that $\Delta_{g}((g):(f))+(f)=A[Y]$. In particular $1 \in \Delta_{g}+(f)$ and $1 \in((g):(f))+(f)$. Then $\Delta_{g}(\tilde{y})=A$ and so we have $g(\tilde{y})$ $\equiv 0 \bmod \Delta_{g}^{2}(\tilde{y})$ a. By Newton Lemma there exist a solution $y$ of $g$ in $A$ such that $y \equiv \tilde{y} \bmod \Delta_{g}(\tilde{y})$ a, i.e. $y \equiv \tilde{y} \bmod a$. Moreover $y$ is a solution for $f$ because $((g):(f))(y) f(y) \subset(g(y))=0$ and $((g):(f))(y)$ contains an invertible element since

$$
A=((g):(f))(\tilde{y}) \subset((g):(f))(y)+\mathfrak{a} .
$$

Clearly the map $\sigma: B \rightarrow A$ induced by $Y \rightarrow y$ lifts $\bar{\sigma}$ Q.E.D. 
A ring morphism $u: A \rightarrow A^{\prime}$ is called algebraically pure if every finite system of polynomial equations over $A$ has a solution in $A$ iff it has one in $A^{\prime}$ (see [ $\left.\mathrm{P}_{1}\right]$, [BNP] for examples and properties). In other words $u$ is algebraically pure if for every finite presentation $A$-algebra $B$ it holds $\operatorname{Hom}_{\mathrm{Alg} A}(B, A) \neq \phi$ iff $\operatorname{Hom}_{\mathrm{Alg} A}\left(B, A^{\prime}\right) \neq \phi$. The following Theorem is close to [BNP] Theorem (5.2).

(3.2) Theorem. Let $(A, \mathfrak{a})$ be a henselian couple and $u: A \rightarrow A^{\prime} a$ regular morphism of noetherian rings. Then the following conditions are equivalent:

i) $A / \mathfrak{a} \otimes_{A} u$ is algebraically pure,

ii) $u$ is algebraically pure.

Proof. ii) $\Rightarrow$ i) is a consequence of $\left[\mathrm{P}_{1}\right]$ Corollary (1.3).

i) $\Rightarrow$ ii) Let $B$ be a finite presentation $A$-algebra and $h: B \rightarrow A^{\prime}$ an $A$-morphism. By Theorem $(2.5),(B, h)$ has a desingularization, i.e. there exist a standard smooth $A$-algebra $B^{\prime}$ and two $A$-morphisms $v: B \rightarrow B^{\prime}$, $w: B^{\prime} \rightarrow A^{\prime}$ such that $w v=h$.

Since $A / \mathfrak{a} \otimes_{A} u$ is algebraically pure and $\operatorname{Hom}_{\mathrm{Alg} A}\left(B^{\prime}, A^{\prime} / \mathfrak{a} A^{\prime}\right)$ contains at least the composite map $B^{\prime} \stackrel{w}{\longrightarrow} A^{\prime} \longrightarrow A^{\prime} / \mathfrak{a} A^{\prime}$ we get $\operatorname{Hom}_{\mathrm{Alg} A}\left(B^{\prime}, A / \mathfrak{a}\right)$ $\neq \phi$. Then $\operatorname{Hom}_{\mathrm{Alg} A}\left(B^{\prime}, A\right) \neq \phi$ by Lemma (3.1). Let $\tilde{w}: B^{\prime} \rightarrow A$ be an $A$ morphism. Then $\operatorname{Hom}_{\Lambda 1 \mathrm{~g} A}(B, A)$ contains at least $\tilde{w} v$.

Q.E.D.

(3.3) Corollary. Theorem (1.3) holds.

Proof. By Theorem (3.2), the morphism $A \rightarrow \hat{A}$ is algebraically pure because $A / \mathfrak{a} \stackrel{\sim}{\longrightarrow} / \mathfrak{a} \hat{A}$ is so. Then $(A, \mathfrak{a})$ satisfies (WAP) and we are ready by the following:

(3.3.1) Lemma. A noetherian couple is an (AP)-couple if it satisfies (WAP).

Proof of Lemma (3.3.1). Suppose that $(A, \mathfrak{a})$ satisfies (WAP) and let $b=\left(b_{1}, \cdots, b_{t}\right)$ be a system of generators for a. Let $f$ be a finite system of polynomial equations over $A, \hat{y}=\left(\hat{y}_{1}, \cdots, \hat{y}_{n}\right)$ a solution of $f$ in the completion $\hat{A}$ and $c$ a positive integer. We can choose in $A$ a system of elements $\tilde{y}=\left(\tilde{y}_{1}, \cdots, \tilde{y}_{n}\right)$ such that $\tilde{y} \equiv \hat{y} \bmod a^{c} \hat{A}$; i.e., there exist in $\hat{A}$ some elements $\hat{z}=\left(\hat{z}_{i s}\right)$ such that

$$
\tilde{y}_{i}=\hat{y}_{i}+\sum_{\substack{s=\left(s_{1}, \ldots, s_{t}\right) \\|s|=c}} \hat{z}_{i s} b_{1}^{s_{1}} \cdots b_{t}^{s_{t}}, \quad 1 \leqslant i \leqslant n .
$$


Then $(\hat{y}, \hat{z})$ is a solution of the following system of equations.

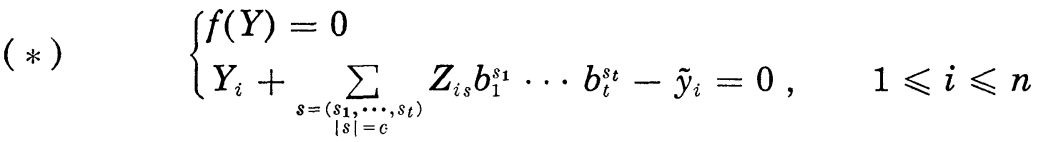

Using (WAP), (*) has a solution $y, z$ in $A$ and thus we have $f(y)=0$ and $y \equiv \tilde{y} \equiv \hat{y} \bmod \mathfrak{a}^{c} \hat{A}$.

Q.E.D.

(3.4) CoRollary. The completion of a factorial excellent henselian local ring is still factorial.

Proof. One knows (see [KMPPR] Ch. V, or [BNP] Propositions (4.7.2), (5.1)) that the completion of a factorial AP-ring is still factorial. Thus it is enough to apply Theorem (1.3).

Q.E.D.

(3.5) Corollary. Let $A$ be an AP-ring. Then the henselization $A\langle X\rangle$, $X=\left(X_{1}, \cdots, X_{n}\right)$ of the couple $(A[X],(X))$ is also an AP-ring.

Proof. When $A$ is a complete local ring, $A\langle X\rangle$ is excellent henselian and the result follows from Theorem (1.3). Otherwise, note that the canoni-

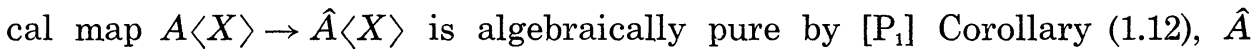
being the completion of $A$. But $\hat{A}\langle X\rangle$ is an AP-ring by Theorem (1.3) and so the map $\hat{A}\langle X\rangle \rightarrow \hat{A} \llbracket X \rrbracket$ is algebraically pure. Then the composite map $A\langle X\rangle \rightarrow \hat{A}\langle X\rangle \rightarrow \hat{A} \llbracket X \rrbracket$ is also algebraically pure and we are ready by Lemma (3.3.1).

Q.E.D.

As we shall see below, Theorem (1.4) is a consequence of the above Corollary. The analytical version of Theorem (1.4) is false by [Ga], the counterexample being given on three variables. Then it was not believable that a result like the above Corollary (and hence Theorem (1.3)) holds. However in [KMPPR] Ch. III appeared a Theorem like Theorem (1.4) in characteristic zero but the proof was wrong. Since in [KMPPR] Ch. IV the quoted "result" had already some nice applications the research continued. A big hope appeared when in $\left[\mathrm{PP}_{2}\right]$ was proved the following result which shows in particular that there exist no counterexamples in three variables for Theorem (1.4).

"Let $A$ be a formal power series ring in two variables over a field and $X$ a variable. Then $A\langle X\rangle$ the henselization of $(A[X],(X))$ is an AP-ring". Recently in [BDL] was showed that $A\left\langle X_{1}, X_{2}, X_{3}\right\rangle$ is an AP-ring, $A$ being like above. 
(3.6) Lemma. Let $A \rightarrow B$ be an algebraically pure morphism, $X=$ $\left(X_{1}, \cdots, X_{r}\right)$ some variables, $A\langle X\rangle, B\langle X\rangle$ the henselization of $(A[X],(X))$, resp. $(B[X],(X)), f$ a finite system of polynomial equations over $A\langle X\rangle$ and $\tilde{y}=\left(\tilde{y}_{1}, \cdots, \tilde{y}_{n}\right) \in B\langle X\rangle^{n}$ a solution of $f$ such that $\tilde{y}_{i} \in B\left\langle X_{1}, \cdots, X_{s_{i}}\right\rangle, 1 \leqslant i$ $\leqslant n$ for some positive integers $s_{i} \leqslant r$. Then there exists a solution $y=$ $\left(y_{1}, \cdots, y_{n}\right)$ of $f$ in $A\langle X\rangle$ such that $y_{i} \in A\left\langle X_{1}, \cdots, X_{s_{i}}\right\rangle, 1 \leqslant i \leqslant n$.

Proof. As $\tilde{y}_{i} \in B\left\langle X_{1}, \cdots, X_{s_{i}}\right\rangle$ there exists an etale neighbourhood of $B\left[X_{1}, \cdots, X_{s_{i}}\right]$ containing $\tilde{y}_{i}$. Thus there exist some systems of polynomials $\tilde{P}=\left(\tilde{P}_{i}\right)_{i}, \tilde{H}=\left(\tilde{H}_{i}\right), \tilde{G}=\left(\tilde{G}_{i}\right)$ from $B\left[X_{1}, \cdots, X_{s_{i}}, T_{i}\right], 1 \leqslant i \leqslant n$ such that for every $i, 1 \leqslant i \leqslant n$

1) $\tilde{P}_{i}$ is monic in $T_{i}$,

2) $\tilde{P}_{i}\left(T_{i}=0, X=0\right)=0$,

3) $\tilde{H}_{i}$ is a multiple of $\partial \tilde{P}_{i} / \partial T_{i}$,

4) $\tilde{H}_{i}\left(T_{i}=0, X=0\right)$ is invertible in $B$,

5) $\tilde{y}_{i}$ can be identified with the image of $\tilde{G}_{i} / \tilde{H}_{i}^{e}$ in $C_{i}:=\left(B\left[X_{1}, \cdots, X_{s}\right.\right.$, $\left.\left.T_{i}\right] / \tilde{P}_{i}\right)_{\tilde{H}_{i}}$ for a certain positive integer $e$.

Denote $\tilde{H}:=\prod_{i=1}^{n} \tilde{H}_{i}, T=\left(T_{1}, \cdots, T_{n}\right), C:=(B[X, T] /(\tilde{P}))_{\tilde{H}}$ and suppose that $\tilde{P}_{i}, \tilde{H}_{i}, \tilde{G}_{i}$ have the following forms

$$
\begin{aligned}
\tilde{P}_{i} & =T_{i}^{t_{i}}+\sum_{k=0}^{t_{i}-1} \sum_{\substack{j=\left(j_{1}, \ldots, j, j\right) \\
0 \leqslant j \mid<\omega}} \tilde{u}_{i j k} X^{j} T_{i}^{k}, \\
\tilde{H}_{i} & =\sum_{k=0}^{\infty} \sum_{0 \leqslant|j|<\omega} \tilde{v}_{i j k} X^{j} T_{i}^{k}, \\
\tilde{G}_{i} & =\sum_{k=0}^{\infty} \sum_{0 \leqslant|j|<\omega} \tilde{w}_{i j k} X^{j} T_{i}^{k},
\end{aligned}
$$

where $\omega$ is a certain positive integer and $\tilde{u}_{i j k}, \tilde{v}_{i j k}, \tilde{w}_{i j k} \in B$ satisfy

6) $\tilde{u}_{i j k}=\tilde{v}_{i j k}=\tilde{w}_{i j k}=0$ if there exist $\lambda, s_{i}<\lambda \leqslant r$ such that $j_{\lambda} \neq 0$, $j=\left(j_{1}, \cdots, j_{r}\right)$.

7) $\tilde{u}_{i \overline{0} 0}=0$ where $\overline{0}=(0, \cdots, 0)$ (see 2))

8) $\tilde{v}_{i \overline{0}}$ is invertible in $B$ (see 4 )), i.e. there exist $\tilde{z}_{i} \in B$ such that $\tilde{v}_{i \overline{0} 0} \tilde{z}_{i}=1$.

Since $f(\tilde{y})=0$ there exist a positive integer $c$ such that

9) $\tilde{H}^{c} F\left(\tilde{H}^{e}, \tilde{G}\right) \subset(\tilde{P})$,

where $F(Y, X)$ is the system of homogeneous polynomials obtained by clearing the denominator of $f(X / Y)$.

In other words 3), 8) and 9) say that $\left\{\tilde{u}_{i j k}, \tilde{v}_{i j k}, \tilde{w}_{i j k}\right\}$ is a part of a solution for a certain system $S$ of polynomials in $\left\{U_{i j k}, V_{i j k}, W_{i j k}\right\}$ and some other variables over $A$. Clearly we can arrange $S$ such that a variable 
$U_{i j k}$ (resp. $V_{i j k}, W_{i j k}$ ) does not enter effectively in $S$ if $\tilde{u}_{i j k}=0$ (resp. $\tilde{v}_{i j k}$ $=0, \tilde{w}_{i j k}=0$ ). Then $S$ has solutions in $B$ iff $f$ has a solution $\tilde{y}$ in $B\langle X\rangle$ which is subject to our hypothesis.

Since the map $A \rightarrow B$ is algebraically pure $S$ has a solution $\left\{u_{i j k}, v_{i j k}\right.$, $\left.w_{i j k}\right\}$ in $A$. Then as we already noticed $f$ must have a solution $y$ which is subject to our Lemma.

Q.E.D.

(3.7) Theorem. Let $A$ be an AP-ring, $\hat{A}$ its completion, $A\langle X\rangle$ the henselization of $(A[X],(X)), X=\left(X_{1}, \cdots, X_{r}\right), f$ a finite system of polynomial equations over $A\langle X\rangle$ and $\hat{y}=\left(\hat{y}_{1}, \cdots, \hat{y}_{n}\right) \in \hat{A} \llbracket X \rrbracket^{n}$ a formal solution of $f$ such that $\hat{y}_{i} \in \hat{A}\left[X_{1}, \cdots, X_{s_{i}}\right], 1 \leqslant i \leqslant n$ for some positive integers $s_{i} \leqslant r$. Then there exists a solution $y=\left(y_{1}, \cdots, y_{n}\right)$ of $f$ in $A\langle X\rangle$ such that $y_{i} \in$ $A\left\langle X_{1}, \cdots, X_{s_{i}}\right\rangle, 1 \leqslant i \leqslant n$.

Proof. By recurrence we may reduce our problem to the case when there exists $t \in\{1, \cdots, n-1\}$ such that $s_{i}=0$ for $i \leqslant t$ and $s_{i}=r$ for $i>t$. As $\hat{A}\langle X\rangle$ is an AP-ring by Corollary (3.5), the system of equations

$$
f\left(\hat{y}_{1}, \cdots, \hat{y}_{t}, Y_{t+1}, \cdots, Y_{n}\right)=0
$$

has a solution $\left(\tilde{y}_{t+1}, \cdots, \tilde{y}_{n}\right)$ in $\hat{A}\langle X\rangle$ because it has one in $\hat{A} \llbracket X \rrbracket$. Applying the above Lemma we can find a solution $y$ in $A\langle X\rangle$ such that $y_{i} \in A$ for $1 \leqslant i \leqslant t$.

Q.E.D.

(3.7.1) Remark. Moreover given a positive integer $c$ we can choose $y$ in Theorem (3.7) such that $y \equiv \hat{y} \bmod (\mathfrak{m}, X)^{c}, \mathfrak{m}$ being the maximal ideal of $A$ (the proof goes like before enlarging the system $f$ like in Lemma (3.3.1)).

(3.7.2) Remark. If $A$ is a reduced AP-ring then by [Ra] Ch. XI, Section 3 Theorem 3 or [KPR] (2.10.2) $A\langle X\rangle$ is exactly the algebraic power series ring in $X$ over $A$, because the formal fibers of an AP-ring are geometrically normal (see $[\mathrm{B}]$ or $[\mathrm{CP}]$ ).

\section{$\S 4$. Ultrapowers and the property of strong approximation}

(4.1) Let $A$ be a ring and $D$ a nonprincipal ultrafilter on $N$-the positive integer set. The ultrapower $A^{*}$ of $A$ with respect to $D$ is a factor ring of $A^{N}$ by the ideal $I_{D}=\left\{\left(x_{n}\right)_{n \in N} \mid\left\{n \in N \mid x_{n}=0\right\} \in D\right\}$ (see [BDLV]). Given a sequence of elements $\left(y_{n}\right)_{n \in N}$ from $A$ we shall denote by $\left[\left(y_{n}\right)_{n \in N}\right]$ $\in A^{*}$ its residue class modulo $\mathrm{I}_{n}$. The map $\varphi: A \rightarrow A^{*}$ given by $x \rightarrow[(x, \cdots$, 
$x, \cdots)]$ is algebraically pure (thus injective) and gives the $A$-algebra structure on $A^{*}$. The map $\varphi$ is flat when $A$ is a noetherian ring.

(4.2) If $A$ is not artinian then $A^{*}$ is not noetherian. But if $(A, \cdots)$ is a noetherian local ring then the separated ring $A_{1}=A^{*} / \bigcap_{n \in N} \mathrm{~m}^{n} A^{*}$ of $A^{*}$ with respect to the m-adic topology is the Cohen $A$-algebra whose residue field is $k^{*}$-the ultrapower of $k=A / \mathrm{m}$ with respect to $D$ (see $\left[\mathrm{P}_{1}\right]$. [BNP]). Prime ideals of $A$ extend to prime ideals of $A^{*}$, the maximal (minimal) ones extends to the maximal (resp. minimal) ones. Let $f$ be a countable system of polynomial equations over $A^{*}$. Then $f$ is compatible in $A^{*}$ iff every finite subsystem of it is compatible in $A^{*}$ (see $\left[\mathrm{P}_{1}\right]$ ).

The following Lemma gives a nonstandard form of the strong approximation property (see [BNP]).

(4.3) Lemma. Let $(A, \mathfrak{a})$ be a noetherian couple. Then the following conditions are equivalent:

i) $(A, \mathfrak{a})$ is $a$ (SAP)-couple,

ii) for every finite system of polynomials $f$ over $A$, for every positive integer $c$ and for every system of elements $\hat{y}$ from $A^{*}$ which form a solution of $f$ modulo $\mathfrak{a}_{s o}=\bigcap_{n \in N} \mathfrak{a}^{n} A^{*}$ there exists a solution $y_{c}$ of $f$ in $A^{*}$ such that $y_{c} \equiv \hat{y} \bmod \mathfrak{a}^{c} A^{*}$,

iii) for every finite type $A$-algebra $B$ for every positive integer $c$ and for every A-morphism $g: B \rightarrow A_{1}=A^{*} / \mathfrak{a}_{\infty}$ there exists an $A$-morphism $h: B \rightarrow A^{*}$ which makes commutative the following diagram

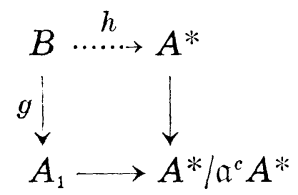

Proof. Clearly ii) $\Leftrightarrow$ iii). The implications i) $\Rightarrow$ ii) are easy extensions of those from [BNP], Theorem (3.7), or Proposition (3.2.1) but we shall prove them.

i) $\Rightarrow$ ii) Let $f, c, \hat{y}$ be like in ii) and $\nu: N \rightarrow N$ the function associated to $f$ by $(\mathrm{SAP})$. Since $f(\hat{y}) \equiv 0 \bmod a^{\nu(c)} A^{*}$, the set

$$
\delta_{c}:=\left\{j \in N \mid f\left(\hat{y}_{j}\right) \equiv 0 \bmod a^{\nu(c)}\right\} \in D
$$

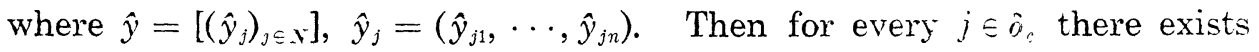
a solution $y_{j}$ of $f$ in $A$ such that $y_{j} \equiv \hat{y}_{j} \bmod a^{c}$. Put $y_{i}=0$ for $j \notin \delta_{c}$ and $y=\left[\left(y_{j}\right)_{j \subseteq x}\right]$. Clearly $f(y)=0$ and $y \equiv \hat{y} \bmod \mathfrak{a}^{c} A^{*}$. 
ii) $\Rightarrow$ i) Suppose that there exist a finite system of polynomials $f$ in $Y=\left(Y_{1}, \cdots, Y_{n}\right)$ over $A$ and a positive integer $c$ such that

(*) For every positive integer $j \in N$ there exists a system of elements $\hat{y}_{j} \in A^{n}$ such that $f\left(\hat{y}_{j}\right) \equiv 0 \bmod \mathfrak{a}^{j}$ but there exists no solution $y_{i}$ of $f$ in $A$ such that $y_{j} \equiv \hat{y}_{i} \bmod \mathfrak{a}^{c}$.

Clearly the system of elements $\hat{y}=\left[\left(\hat{y}_{j}\right)_{j \in N}\right] \in A^{* n}$ satisfies $f(\hat{y}) \equiv 0$ $\bmod a^{t} A^{*}$ for every $t \in N$, i.e. $\hat{y}$ form a solution of $f$ modulo $a_{\infty}$. By ii) there exists a solution $y=\left[\left(y_{j}\right)_{j \in N}\right]$ of $f$ in $A^{*}$ such that $y \equiv \hat{y} \bmod \mathfrak{a}^{c} A^{*}$. Then the set

$$
\delta=\left\{j \in N \mid f\left(y_{j}\right)=0, y_{j} \equiv \hat{y}_{j} \bmod \mathfrak{a}^{c}\right\}
$$

belongs to $D$. Thus $\delta$ is nonempty. Take a $j \in \delta$. Then $y_{j}$ contradicts $(*)$.

Q.E.D.

(4.4) Theorem. Let $(A, \mathfrak{a})$ be a noetherian henselian couple and $A^{*}$ the ultrapower of $A$ with respect to a nonprincipal ultrafilter on $N$. Suppose that the composite map $A \rightarrow A^{*} \rightarrow A_{1}=A^{*} / \cap_{n \in N} \mathfrak{a}^{n} A^{*}$ is a filtered inductive limit of standard smooth A-morphisms. Then $(A, \mathfrak{a})$ is a (SAP)-couple.

Proof. Let $B$ be a finite type $A$-algebra $c$ a positive integer and $g$ : $B \rightarrow A_{1}$ an $A$-morphism. By hypothesis there exist a standard smooth $A$ algebra $B^{\prime}$ and two $A$-morphisms $v: B \rightarrow B^{\prime}, w: B^{\prime} \rightarrow A_{1}$ such that $w v=g$. Since $\left(A^{*}, \mathfrak{a} A^{*}\right)$ is a henselian couple $((A, \mathfrak{a})$ being so) the composite map $B^{\prime} \stackrel{w}{\longrightarrow} A_{1} \longrightarrow A^{*} / \mathfrak{a}^{c} A^{*}$ can be lifted to an $A$-morphism $\tilde{w}: B^{\prime} \rightarrow A^{*}$ by Lemma (3.1). Then $h:=\tilde{w} v$ makes commutative the diagram from Lemma (4.3) and we are ready.

Q.E.D.

(4.5) CoRollary. An excellent henselian local ring has the property of strong approximation.

Proof. If $(A, \mathfrak{a})$ is noetherian local then $A_{1}$ is a formally smooth $A$ algebra (see (4.1)). By $\left[\mathrm{An}_{2}\right]$, or [BR] the map $A \rightarrow A_{1}$ is regular when $A$ is excellent. Now the result is a consequence of Theorem (2.5) and (4.4).

Q.E.D.

(4.6) Remark. Using Theorem (4.4) note that Question (1.6) has a positive answer for couples $(R, I)$ such that $R / I$ is excellent if for every excellent ring $A$ and every $n \in N$ the morphism $A \llbracket X \rrbracket \rightarrow A^{*} \llbracket X \rrbracket, X=\left(X_{1}\right.$, $\left.\cdots, X_{n}\right)$ is a filtered inductive limit of standard smooth $A \llbracket X \rrbracket$-morphisms (when $n=0$ and $A$ is a discrete valuation ring containing a field of characteristic zero this was proved in $\left.\left[\mathrm{P}_{5}\right]\right)$. 
(4.7) Corollary (Elkik [E]). Let $(A, \mathfrak{m})$ be a noetherian henselian local ring and $f$ a finite system of polynomial equations over $A$ in $Y=\left(Y_{1}\right.$, $\left.\cdots, Y_{n}\right)$. Then there exists a function $\nu: N \rightarrow N$ with the following property:

"For every positive integer $c$ and for every system of elements $\tilde{y} \in A^{\text {" }}$ such that

i) $\mathfrak{n}=\sqrt{ } H_{f}(\bar{y})$

ii) $f(\tilde{y}) \equiv 0 \bmod m^{\nu(c)}$

there exists a solution $y$ of $f$ in A such that $y \equiv \tilde{y} \bmod : \mathrm{I}^{\prime \prime}$.

Proof. Let $\hat{A}$ be the completion of $A$ and $\nu: N \rightarrow N$ the function associated to $f$ over $\hat{A}$ by (SAP), $\hat{A}$ having (SAP) by Corollary (4.5). Let $c, \tilde{y} \in A^{n}$ be like above. Then there exists a solution $\hat{\theta}$ of $f$ in $\hat{A}$ such that $\hat{y} \equiv \tilde{y} \bmod \mathfrak{m}^{c} \hat{A}$. Take $B:=A[Y] /(f)$ and let $g$ be the $A$-morphism $B \rightarrow \hat{A}$ given by $Y \rightarrow \hat{y}$. We have

$$
\begin{aligned}
& g\left(H_{B / A}\right)=H_{f}(\hat{y}) \text { and } \\
& H_{f}(\hat{y}) \hat{A} \equiv H_{f}(\tilde{y}) \hat{A} \bmod m^{c} \hat{A} .
\end{aligned}
$$

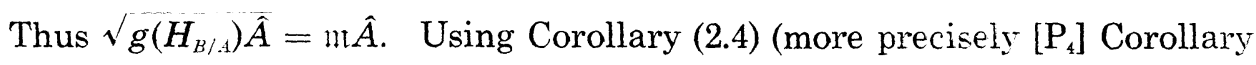
(5.3)) there exist a standard smooth $A$-algebra $B^{\prime}$ and two $A$-morphisms $v: B \rightarrow B^{\prime}, w: B^{\prime} \rightarrow \hat{A}$ such that $w v=g$. By Lemma (3.1) the composite map $B^{\prime} \stackrel{w}{\longrightarrow} \hat{A} \longrightarrow \hat{A} / \mathfrak{m}^{c} \hat{A} \cong A / \mathfrak{m}^{c}$ can be lifted to a map $u^{\prime}: B^{\prime} \rightarrow A$. Take $y$ to be the image of $Y$ by $w^{\prime} v$ and we are ready.

Q.E.D.

(4.7.1) Remark. A positive answer to Question (1.6) would extend the above Corollary for couples as it was already proved in [E].

\section{§5. A partial extension of Cohen-algebras}

(5.1) Lemma. Let $(A, \mathfrak{a})$ be a couple and $\bar{B}$ a standard smooth $\bar{A}:=$ $A / \mathfrak{a}$-algebra. Then there exist a standard smooth A-algebra $B$ such that $\bar{B} \cong B / \mathfrak{a} B$.

Proof. By $(2.1)$ we have $\bar{B} \cong(\bar{A}[Y] /(\bar{g}))_{\bar{P}}, \quad Y=\left(Y_{i}, \cdots, Y_{n}\right), \bar{g}=\left(\bar{g}_{i}\right.$, $\left.\ldots, \bar{g}_{r}\right), \bar{P} \in \mathcal{L}_{\bar{g}}$. Then there exist some $r \times r$-minors $\left\{\bar{M}_{i}\right\}_{i-\cdots s}$ of the matrix $(\hat{o} \bar{g} / \partial Y)$ and some polynomials $\bar{h}=\left(\bar{h}_{1}, \cdots, \bar{h}_{s}\right)$ from $\bar{A}[Y]$ such that $\bar{P}=$ $\sum_{i=1}^{s} \bar{h}_{i} \bar{M}_{i}$. Lift $\bar{g}, \bar{h}$ to some polynomials $g, h$ over $A$ and let $\left\{M_{i}\right\}_{i}$ be the $r \times r$-minors of $(\partial g / \partial Y)$ corresponding to $\left\{\bar{M}_{i}\right\}$. Clearly $P:=\sum_{i=1}^{s} h_{i} M_{i}$ lifts $\bar{P}$ and $B=(A[Y] /(g))_{P}$ works. $\quad$ Q.E.D.

(5.2) Lemma. Let $(A, \mathfrak{a})$ be a couple and $\bar{B}$ a filtered inductive limit 
of a countable set of standard smooth $\bar{A}:=A / \mathfrak{a}$-algebras. Then there exist a flat A-algebra $B$ such that

i) $\bar{B} \cong B$ u $B$ as $\bar{A}$-algebras,

ii) $\boldsymbol{B}$ is a filtered inductive limit of a countable set of standard smooth A.algebras.

Proof. Suppose that $\bar{B} \cong \lim _{i \in N}\left(\bar{B}_{j}, \bar{\varphi}_{j}\right), \bar{\varphi}_{j}: \bar{B}_{j} \rightarrow \bar{B}_{j+1}$, where $\left\{\bar{B}_{j}\right\}$ are standard smooth over $A$. By Lemma (5.1) there exist some standard smooth $A$-algebras $\left\{B_{j} j \in N\right\}$ such that $\bar{B}_{j}=B_{j} / \mathfrak{a} B_{j}$. For every $j \in N$, let $B_{j}^{h}$ be the henselization of $B_{j}$ at $\mathfrak{a} B_{j}$. By Lemma (3.1) the composite map $B_{j} \rightarrow$ $\bar{B}_{j} \stackrel{\varphi_{j}}{\longrightarrow} \bar{B}_{j+1} \cong B_{j+1}^{i} / a B_{j+1}^{h}$ can be lifted to an $A$-morphism $\psi_{j}: B_{j} \rightarrow B_{j+1}^{h}$. Then changing (by recurrence) $\left\{B_{j}\right\}_{j}$ up to some etale neighborhoods of them we can also suppose that there exist some liftings $\varphi_{j}: B_{j} \rightarrow B_{j+1}$, $j \in N$ of $\left\{\bar{\varphi}_{j}\right\}$. Clearly the filtered inductive limit $B$ of $\left\{B_{j}, \varphi_{j}\right\}_{j=N}$ is subject to i), ii). Since $\left\{B_{i}\right\}$ are flat over $A, B$ is flat too.

Q.E.D.

(5.3) Corollary. Let $(A, \mathfrak{a})$ be a couple and $\bar{B}$ a filtered inductive limit of a countable set of standard smooth $\bar{A}:=A / a$-algebras. Suppose that it is a nil-ideal. Then there exists a flat A-algebra $B$ such that

i) $\bar{B} \cong B \backsim B$ as $\bar{A}$-algebras,

ii) $\boldsymbol{B}$ is a filtered inductive limit of a countable set of standard smooth A-algebras.

Moreover if $B^{\prime}$ is another flat A-algebra such that $\bar{B}^{\vdots} \cong B^{\prime} / \mathfrak{a} B^{\prime}$ then there exists an $A$-isomorphism $B \rightarrow B^{\prime}$ lifting the composite isomorphism $B_{/}^{\prime} \mathfrak{B} \cong \bar{B} \cong B^{\prime} / \mathrm{r} B^{\prime}$; in particular $B^{\prime}$ is also subject to ii).

For the proof apply the above Lemma, the unicity being given like in [EGA] Part 1 (19, 7.1.5).

Q.E.D.

(5.4) Corollary. Let $A \rightarrow A^{\prime}$ be a flat ring morphism and $\mathfrak{a} \subset A$ a nil-ideal. Then the following conditions are equivalent

1) $A^{\prime}$ is a filtered inductive limit of a countable set of standard smooth A-algebras,

2) $\boldsymbol{A}^{\prime} / \mathrm{a} \boldsymbol{A}^{\prime}$ is a filtered inductive limit of a countable set of standard smooth $\boldsymbol{A} / \mathfrak{a}$-algebras.

Proof. $\mathrm{By}$ base change we have 1 ) $\Rightarrow 2$ ). If 2 ) holds there exist a flat $A$-algebra $B$ which is subject to i), ii) from Corollary (5.3). By unicity $B \cong A^{\prime}$ and so 1) holds.

Q.E.D. 
(5.5) Theorem. Let $(A, a)$ be a noetherian couple and $A / a \rightarrow \bar{B}$ a regular morphism of noetherian rings. Suppose that $\bar{B}$ is countable generated over $\bar{A}:=A / \mathfrak{a}$. Then there exist a noetherian flat $A$-algebra $B$ such that

i) $\bar{B} \cong B / \mathfrak{a} B$ as $\bar{A}$-algebras,

ii) $B$ is complete at $a B$.

Proof. By Lemma (5.2) and Theorem (2.5) there exists a flat $A$-algebra $C$ such that $C / \mathfrak{a} C \cong \bar{B}$. Since $\bar{B}$ is noetherian and a is finitely generated the completion $B$ of $C$ at $\mathfrak{a} C$ is also noetherian ([ZS] VIII $\S 3$, p. 260). By [ZS] VIII Theorem 5 we have $B / \mathfrak{a}^{n} B \cong C / \mathfrak{a}^{n} C$ for every $n \in N$.

Thus $B / \mathfrak{a}^{n} B$ are flat $A / \mathfrak{a}^{n}$-algebras, $n \in N$ and so $B$ is flat over $A$ by [M] (20.C).

Q.E.D.

(5.6) Remark. i) When $\bar{B}$ is local then according to [EGA], part 1, (19.7.1.5) $B$ is unique, i.e. if $B^{\prime}$ is another noetherian flat $A$-algebra satisfying i), ii) from Theorem (5.5) then there exists an $A$-isomorphism $B \rightarrow B^{\prime}$ lifting the composite isomorphism $B / \mathfrak{a} B \cong \bar{B} \cong B^{\prime} / \mathfrak{a} B^{\prime}$.

ii) The Theorem (5.5) is a partial positive answer to [EGA] part 1 (19.7.3).

\section{§6. Some remarks on field extensions of characteristic $>0$}

In this section $k \subset K$ is always a field extension of characteristic $p>0$ and $K_{n}:=k\left(K^{p^{n}}\right), n$ being a nonnegative integer.

(6.1) Lemma. Let $x=\left(x_{i}\right)_{i \Subset I}, y=\left(y_{1}, \cdots, y_{t}\right)$ be some elements from $k$ and $r$ a positive integer. Suppose that:

i) $x \cup y$ form a p-basis in $k$ over $k^{p}$,

ii) $x$ form in $K$ a system p-independent over $K^{p}$,

iii) there exist a finite subset $J \subset I$ and some elements $\left\{\boldsymbol{z}_{u e} \mid 1 \leqslant u \leqslant t\right.$, $\left.e=\left(e_{j}\right)_{j \in J}, 0 \leqslant e_{j}<p^{\prime}\right\}$ from $K$ such that

$$
y_{u}=\sum_{\substack{e=\left\{e_{j}\right) \\ 0 \leqslant e_{j} \backslash p^{r}}} \boldsymbol{z}_{\eta_{u} e^{p^{r}}}\left(\prod_{j \in J} x_{j}^{e_{j}}\right), \quad u=1, \cdots, t .
$$

Then $\operatorname{rk} \Gamma_{K_{r-1 / k}}=t . \quad$ Moreover if $\operatorname{rk} \Gamma_{K_{r} / k}=t$ then $z_{u e} \in K^{\mu}(x)$ for all $u, e$.

Proof. By iii) we have $y_{u} \in K^{p r}(x)$ and so $\mathrm{rk} \Gamma_{K_{r-1 / k}} \geqslant t$ (see i)), the opposite inequality being a consequence of ii).

Suppose that $\mathrm{rk} \Gamma_{K_{r} / k}=t$. Then $y_{u}$ must be in $K_{r}^{p}(x)$ because $x$ is $p$ independent in $K_{r}$ over $K_{r}^{p}$ and $x \cup y$ form a $p$-basis in $k$ over $k^{p}$. We claim that $y_{u}$ belongs even to $K^{p^{r+1}}(x)$. Indeed we have $k=k^{p^{r}}(x, y)$ and 
so $K_{r}^{p}(x)=K^{p^{r+1}}\left(k^{p}(x)\right)=K^{p r+1}\left(y^{p}, x\right)$. Then $y_{u} \in K^{p^{r+1}}\left(y^{p}, x\right)$ and by recurrence we prove our claim.

Consequently there exists an expression like (6.1.1) for some elements $\boldsymbol{z}_{u e}^{\prime} \in K^{p}(x)$. Since $\left\{\boldsymbol{z}_{u e}\right\}$ are given in an unique way by (6.1.1) we get $\boldsymbol{z}_{u e}$ $\in K^{p}(x)$.

Q.E.D.

(6.2) Lemma. Let $r$ be a nonnegative integer. Suppose that for every integer $i, 0 \leqslant i \leqslant r$ the extension $k \subset K_{i}$ has

$$
t=\mathrm{rk}_{K_{i}} \Gamma_{K_{i} / k}=\text { constant }<\infty, t>0 .
$$

Then there exist a finite type field subextension $F / k$ of $K / k$ such that

i) every field subextension $E / k$ of $K / k$ satisfies

$$
\operatorname{rk} \Gamma_{k\left(E^{\left.p^{i}\right) / k}\right.}=t, \quad 0 \leqslant i \leqslant r
$$

iff $E \supset F$,

ii) every $p$-basis of $F$ over $k\left(F^{p}\right)$ generates $F$ over $k$.

Proof. Let $x=\left(x_{i}\right)_{i \in I}$ be a maximal system of elements from $k$ which is $p$-independent in $K$ over $K^{p}$. By hypothesis there exists a system of elements $y=\left(y_{1}, \cdots, y_{t}\right)$ from $k$ such that $x \cup y$ form a $p$-basis of $k$ over $k^{p}$. Using the maximality of $x$ we get $y_{u} \in K^{p}(x), 1 \leqslant u \leqslant t$.

By induction on $s, 0 \leqslant s \leqslant r$, we shall find a finite subset $J_{s} \subset I$ and some elements $z_{s}=\left(z_{\text {sues }}\right)$ from $K$ such that

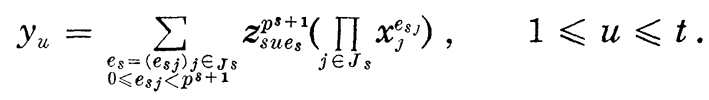

When $s=0$ this follows trivially because $y_{u} \in K^{p}(x)$. Suppose we are ready for $s<r$. Then $z_{\text {sues }} \in K^{p}(x)$, for all $u$, $e_{s}$ by Lemma (6.1).

It follows $y_{u} \in K^{p^{s+2}}(x)$ and so we find $J_{s+1} \supset J_{s}$ and $z_{s+1}$ satisfying our induction.

Take $F=k\left(z_{r}\right)$ and let $E$ be a subfield of $K$ containing $k$. Then the following statements are equivalent:

1) $\operatorname{rk} \Gamma_{k\left(E^{\left.p^{i}\right) / k}\right.}=t, 0 \leqslant i \leqslant r$

2) $y_{u} \in E^{p^{r+1}}(x)$.

Indeed 2$) \Rightarrow 1$ ) holds by Lemma (6.1) and 1) $\Rightarrow 2$ ) goes like above. Since $z_{s}$ is uniquely determined by (6.2.1) we get $y_{u} \in E^{p^{r+1}}(x)$ iff $z_{\text {ruer }} \in E$ for all $e_{r}$, i.e. i) holds.

Now, let $w$ be a $p$-base of $F$ over $k\left(F^{p}\right)$ and $L=k(w)$. Then $F=L\left(F^{p}\right)$ and consequently $F$ is a separable finite extension of $L$ ( $F$ is by construc- 
tion a finite type extension of $L)$. Thus $K\left(L^{p i}\right) \subset k\left(F^{p^{i}}\right)$ is a separable (finite) extension and it follows

$$
\operatorname{rk} \Gamma_{k\left(L p^{p}\right) / k}=t, \quad 0 \leqslant i \leqslant r
$$

By i) we get $L=F$.

Q.E.D.

(6.3) Theorem. Suppose that for every nonnegative integer $n$, the extension $k \subset K_{n}$ has

$$
t:=\operatorname{rk}_{k_{n}} \Gamma_{K_{n} / k}=\text { constant }<\infty, t>0 .
$$

Then there exists an increasing chain of subfields of $K$ containing $k$

$$
F_{0} \subset F_{1} \subset \cdots F_{n} \subset \cdots
$$

such that for every nonnegative integer $n$

i) $F_{n} / k$ is a finite type extension and $\mathrm{rk} \Gamma_{F_{n} / k}=t$,

ii) every $p$-basis of $F_{n}$ over $k\left(F_{n}^{p}\right)$ generates $F_{n}$ over $k$,

iii) $F_{n} \subset k\left(F_{n+1}^{p}\right)$.

Moreover the union $L$ of $\left(F_{n}\right)_{n}$ holds

1) $L=k\left(L^{p}\right)$ and $\operatorname{rk} \Gamma_{L / k}=t$,

2) $K / L$ is a separable field extension.

Proof. For every nonnegative integer $n$ let $F_{n}$ be the field given by Lemma (6.2) for $K / k$ and $r=n$. Then $F_{n}$ satisfies i), ii) and for every field subextension $E / k$ of $K / k$ we have $\operatorname{rk} \Gamma_{k\left(E^{\left.p^{i}\right) / k}\right.}=t, 0 \leqslant i \leqslant n$ iff $E \supset F_{n}$. Taking $E:=k\left(F_{n+1}^{p}\right)$ we get $E \supset F_{n}$, i.e. iii).

Now clearly $\Gamma_{L / K}$ is the filtered inductive limit of the following chain of injective maps

$$
\Gamma_{F_{0} / k} \otimes L \longrightarrow \Gamma_{F_{1 / k}} \otimes L \longrightarrow \cdots \longrightarrow \Gamma_{F^{\prime} / k} \otimes L \longrightarrow
$$

which are actually isomorphisms by i). Thus $\operatorname{rk} \Gamma_{L / k}=t$ and by iii) it holds 1). Then $\Omega_{L / k}=0$ and the first map from the following exact sequence

$$
\Gamma_{L / k} \otimes K \longrightarrow \Gamma_{K / k} \longrightarrow \Gamma_{K / L} \longrightarrow \Omega_{L / k} \otimes K=0
$$

is isomorphism. Consequently $\Gamma_{K / L}=0$, i.e. 2) holds.

Q.E.D.

(6.3.1) Remark. The hypothesis of the above Theorem are fulfilled for example when $K / k$ is an unramified extension (i.e. $K=k\left(K^{p}\right)$ ), and $\operatorname{rk} \Omega_{k}<\infty$. 


\section{§. Desingularization on a special case}

In this section $(R, m)$ is a formally smooth noetherian local algebra over a field $k$ of characteristic $p>0, K:=R / \mathfrak{m}$ and $K_{n}:=k\left(K^{p^{n}}\right), n$ being an integer $\geqslant 0$. Note that $\operatorname{rk} \Gamma_{K / k} \leqslant d:=\operatorname{dim} R$. Constantly we assume that $K / k$ is not separable.

(7.1) Lemma. Let $x=\left(x_{1}, \cdots, x_{s}\right)$ be a system of elements from $R$. Suppose that the residue $\bar{x}$ of $x \bmod \mathfrak{m}$ form a p-basis of $E:=k(\bar{x})$ over $k\left(E^{p}\right)$. Then:

i) the canonical map $u: k[X] \rightarrow R, X=\left(X_{1}, \cdots, X_{s}\right) \sim x$ is flat,

ii) the height of the prime ideal $q:=u^{-1} \mathrm{~m}$ is $t:=\operatorname{rk} \Gamma_{E / k} \leqslant d$

iii) $R / u(q) R$ is a regular local ring of dimension $d-t$.

Proof. $\mathrm{S}:=k[X]_{q}$ is a smooth local $k$-algebra of residue field $E$. Using a criterion of formally smoothness $\left[\mathrm{An}_{1}\right]$ we get $H_{1}(k, R,-)=H_{1}(k, S,-)$ $=0$. Consider the following commutative diagram

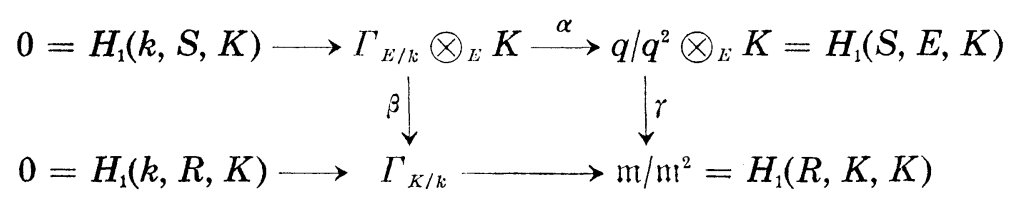

where the top (bottom) exact sequence is a part of the Jacobi-Zariski sequence written for $k \hookrightarrow S \rightarrow E, K$ (resp. $k \hookrightarrow R \rightarrow K, K$ ) and $\gamma, \beta$ are induced by $u$. The map $\beta$ appears in the following exact sequence (see [M] (39. A))

$$
0 \longrightarrow I_{E / k} \otimes_{E} K \stackrel{\beta}{\longrightarrow} I_{K / k}^{\prime} \longrightarrow \Gamma_{K / E} \longrightarrow \cdots
$$

and so it is injective. By Cartier's equality we get $t=s-\operatorname{tr} \operatorname{deg}_{k} E$ and so ht $q=t$. Thus $\operatorname{dim} q S / q^{2} S=t$ and consequently $\alpha$ is bijective. Hence $\gamma$ is injective, i.e. $u$ maps injectively a regular system of parameters from $S$ in a part of a regular system of parameters from $R$. Thus iii) and i) hold (see [M] (36. B)).

Q.E.D.

(7.2) Lemma. Suppose that $(R, \mathfrak{m})$ is complete in its $\mathfrak{m}$-adic topology and for every nonnegatice integer $n$, the extension $k \subset K_{n}$ has

$$
t:=\mathrm{rk} \Gamma_{K_{n} / k}=\text { constant } .
$$

Then there exists a formally smooth noetherian local sub-k-algebra $\left(R^{\prime}, \mathrm{m}^{\prime}\right)$ of $(R, \mathfrak{m})$ such that: 
i) $\mathfrak{m}^{\prime} R=\mathfrak{m}$ and the residual extension $R^{\prime} / \mathfrak{m}^{\prime} \subseteq K$ is separable,

ii) the inclusion $R^{\prime} \subseteq R$ is formally smooth

iii) $R^{\prime}$ is a filtered inductive limit of localizations of polynomial $k$ algebras.

Proof. Let

$$
F_{0} \subset F_{1} \subset \cdots F_{n} \subset \cdots
$$

be the chain of subfields of $K$ given by Theorem (6.3). For every $n$ choose a $p$-basis $\bar{x}_{n}=\left(\bar{x}_{n 1}, \cdots, \bar{x}_{n r_{n}}\right)$ of $F_{n}$ over $k\left(F_{n}^{p}\right)$. Fix $n$. We have $F_{n} \subset k\left(F_{n+1}^{p}\right)$ and $F_{n+1}=k\left(\bar{x}_{n+1}\right)$ and so there exist a system of polynomials $G_{n}=\left(G_{n 1}\right.$, $\left.\cdots, G_{n r_{n}}\right), H_{n}$ from $k\left[X_{n+1}^{p}\right], X_{n+1}=\left(X_{n+1,1}, \cdots, X_{n+1, r_{n+1}}\right)$ such that $H_{n}\left(\bar{x}_{n+1}\right)$ $\neq 0$ and $\bar{x}_{n}=\left(G_{n} / H_{n}\right)\left(\bar{x}_{n+1}\right)$. Denote by $q_{n}$ the kernel of the map $k\left[X_{n}\right] \rightarrow K$ given by $X_{n} \sim \bar{x}_{n}$. Clearly $H_{n} \notin q_{n}$.

Put $S_{n}:=k\left[X_{n}\right]_{q_{n}}, n \geqslant 0$ and let $\varphi_{n}: S_{n} \rightarrow S_{n+1}$ be the $k$-morphism given by $X_{n} \leadsto G_{n} / H_{n}$. Applying Lemma (7.1) to $G_{n} / H_{n}, S_{n+1}$ we get

1) $\left.\varphi_{n}\right|_{k\left[X_{n}\right]}$ flat and so $\varphi_{n}$ is flat too,

2) the height of $q_{n} S_{n}=\varphi_{n}^{-1}\left(q_{n+1} S_{n+1}\right)$ is $t$,

3) $S_{n+1} / \varphi_{n}\left(q_{n}\right) S_{n+1}$ is a field and so $\varphi_{n}\left(q_{n}\right) S_{n+1}=q_{n+1} S_{n+1}$.

Then the ring $S=\underline{\lim }_{n}\left(S_{n}, \varphi_{n}\right)$ is a noetherian local ring of dimension $t$ (see 1)-3), $S_{n}$ being noetherian). Moreover $S$ is a formally smooth $k$-algebra as shows the following:

(7.2.1) Lemma. A filtered inductive limit of formally smooth noetherian local k-algebras is formally smooth too if it is noetherian.

The union $L=\bigcup_{n \geqslant 0} F_{n}$ is the residue field of $S$ and the inclusion $L \subset K$ can be lifted to a $k$-morphism $g: S \rightarrow R, R$ being complete. Using again Lemma (7.1) we get for every $n \geqslant 0$ that

4) $\left.g\right|_{S_{n}}$ is faithfully flat,

5) $g\left(q_{n} S\right) R$ is a prime ideal of height $t$,

6) $R / g\left(q_{n} S\right) R$ is a regular local ring of dimension $d-t$.

Then $g$ is faithfully flat and the maximal ideal $q$ of $S$ holds

7) $R / g(q) R$ is a regular local ring of dimension $d-t$, because as above $g\left(q_{n} S\right) R=g\left(q_{n+1} S\right) R$. Choose a system of $(d-t)$-elements $y$ from $R$ inducing a regular system of parameters in $R / g(q) R$. Take $R^{\prime}:=S[Y]_{(q, Y)}, Y=\left(Y_{1}, \cdots, Y_{d-t}\right), m^{\prime}=(q, Y) R^{\prime}$ and let $h: R^{\prime} \rightarrow R$ be the map extending $g$ by $Y \sim y$. Clearly $\mathfrak{m}^{\prime} R=\mathfrak{m}$ by construction. Since $K / L$ is a separable extension (see Theorem (6.3)) we note that i) holds. 
By [M] (36. B] $h$ is flat, even formally smooth (see [EGA] part 1, (19.7.1)) and by construction we have also iii) fulfilled.

Proof of Lemma (7.2.1). Let $A=\lim _{i \in \underline{I}} A_{i}$ be a filtered inductive limit of formally smooth noetherian local $k$-algebras and $k^{\prime}$ a finite field $p$-extension of $k$. Suppose that $A$ is noetherian. Then $A_{i}^{\prime}:=k^{\prime} \otimes_{k} A_{i}, i \in I$ are regular local rings and $A^{\prime}:=k^{\prime} \otimes_{k} A$ is a noetherian ring. Since $A^{\prime} \simeq \lim _{i \in I} A_{i}^{\prime}$ we note that $A^{\prime}$ is a regular local ring (like in the proof of Theorem (2.5)). Thus $A$ is a geometrically regular $k$-algebra and so $A$ is a formally smooth $k$-algebra (see [EGA] Part 1 (22.5.8))

Q.E.D.

The main result of this section is the following

(7.3) TheOREM. Let $B$ be a finite type k-algebra and $f: B \rightarrow R$ such that $\mathfrak{m}=\sqrt{ } f\left(H_{B / k}\right) R$. Then the couple $(B, f)$ has a desingularization with respect to $k \rightarrow R$.

Proof. First consider a particular case:

Step 1. $t:=\operatorname{rk} \Gamma_{K_{n / k}}=$ constant for every nonnegative integer $n$.

Let $(\hat{R}, \hat{\mathrm{m}})$ be the completion of $R$ in its m-adic topology. Then $\hat{R}$ is a formally smooth $k$-algebra and by Lemma (7.2) there exist a noetherian local sub- $k$-algebra $\left(R^{\prime}, \mathfrak{m}^{\prime}\right)$ of $(\hat{R}, \hat{\mathfrak{m}})$ such that

1) $\mathfrak{m}^{\prime} \hat{R}=\hat{\mathrm{n}}$ and the residual extension $R^{\prime} / \mathfrak{m}^{\prime} G K$ is separable,

2) $R^{\prime}$ is a filtered inductive limit of standard smooth $k$-algebras.

Take $\tilde{B}=R^{\prime} \otimes_{k} B$ and let $\tilde{f}: \tilde{B} \rightarrow \hat{R}$ be the map given by the composite maps

$$
\hat{f}: B \stackrel{f}{\longrightarrow} R \longrightarrow \hat{R}, \quad \hat{h}: R^{\prime} \longrightarrow \hat{R} .
$$

Using $\left[\mathrm{P}_{4}\right]$ Desingularization Lemma $(\tilde{B}, \tilde{f})$ has a desingularization with respect to $\hat{h}$ (note that $H_{\tilde{B} / R^{\prime}}=\left(H_{B / k}\right) \tilde{B}$ ). Since $R^{\prime}$ is a filtered inductive limit of standard smooth $k$-algebras, $(B, \hat{f})$ has also a desingularization with respect to $k \rightarrow \hat{R}$, as shows the following elementary Lemma:

(7.3.1) Lemma. Let $u: A \rightarrow A^{\prime}, v: A^{\prime} \rightarrow A^{\prime \prime}$ be two morphisms of noetherian rings, $B$ a finite type $A$-algebra, $f: B \rightarrow A^{\prime \prime}$ an $A$-morphism, $B^{\prime}:=$ $A^{\prime} \otimes_{A} B$ and $f^{\prime}: B^{\prime} \rightarrow A^{\prime \prime}$ the map given by $v, f$. Then $(B, f)$ has a desingularization with respect to $v u$ if $\left(B^{\prime}, f^{\prime}\right)$ has a desingularization with respect to $v$ and $A^{\prime}$ is a filtered inductive limit of standard smooth A-algebras.

Remains now only to note the following Lemma (its proof is given later). 
(7.3.2) Lemma. ( $B, f)$ has a desingularization with respect to $k \longrightarrow R$ iff $(B, \hat{f})$ has a desingularization with respect to $k=\hat{R}$.

Step 2. General case

The decreasing inequalities

$$
\operatorname{rk} \Gamma_{K / h} \geqslant \operatorname{rk} \Gamma_{K_{1} / k} \geqslant \cdots \geqslant \operatorname{rk} \Gamma_{K_{n} / k} \geqslant \cdots
$$

must stop for a sufficiently high integer $n$. Thus there exists a positive integer $N$ such that

$$
t:=\operatorname{rk} \Gamma_{K_{n} / h}=\text { constant for all } n \geqslant N .
$$

In other words the module of imperfection rank of the extensions $k^{1 / p^{x}} \subset$ $k^{1 / p^{N}}\left(K^{p^{n-N}}\right)$ is constant for every $n \geqslant N$. Choose a finite field subextension $\tilde{k} / k$ of $k^{1 / p^{N}} / k$ such that $\operatorname{rk} \Gamma_{\tilde{k}(K) / \tilde{k}}=t$. Then we have $\operatorname{rk} \Gamma_{\tilde{k}\left(K^{p^{n}}\right) / \tilde{k}}=t$ for every nonnegative integer $n$.

Put $\tilde{K}:=\tilde{k}(K), \tilde{R}:=\tilde{k} \otimes_{k} R, \tilde{B}:=\tilde{k} \otimes_{k} B, \tilde{f}:=\tilde{k} \otimes_{k} f$. By [R] $\tilde{R}$ is still a noetherian formally smooth local $\tilde{k}$-algebra. Clearly $H_{\tilde{B} / \tilde{k}} \supseteq\left(H_{B / k}\right) \tilde{B}$. Now the residue field $\tilde{K}$ of $\tilde{R}$ satisfies the conditions from Step 1 and so like there we note that $(\tilde{B}, \tilde{f})$ has a desingularization with respect to $\tilde{k} \rightarrow \tilde{R}$. We are ready using the following result which is the subject of Section 10.

(7.4) Lemma. Let $u: A \rightarrow A^{\prime}$ be a morphism of noetherian rings, $B$ a finite type A-algebra, $f: B \rightarrow A^{\prime}$ an A-morphism and $\tilde{A}$ a finite free A-algebra. Then $(B, f)$ has a desingularization with respect to $u$ if $\left(\tilde{A} \otimes_{A} B, \tilde{A} \otimes_{A} f\right)$ has a desingularization with respect to $\tilde{A} \otimes_{A} u$.

(7.5) Proof of Lemma (7.3.2). Let $z$ be a regular system of parameters from $R, T:=k[z]$ and $g$ the inclusion $T \hookrightarrow R$. Clearly $g$ is flat (apply e.g. [M] (36. B])). Suppose that $(B, \hat{f})$ has a desingularization with respect to $k \hookrightarrow \hat{R}$, i.e. there exist a standard smooth $k$-algebra $B^{\prime}$ and two $k$ morphisms $v: B \rightarrow B^{\prime}, \hat{w}: B^{\prime} \rightarrow \hat{R}$ such that $\hat{w} v=\hat{f}$. Let $n$ be a positive integer, $\quad \bar{T}:=T /(z T)^{n}, \quad \bar{B}:=\bar{T} \otimes_{k} B, \quad \bar{B}^{\prime}:=\bar{T} \otimes_{k} B^{\prime}, \quad \bar{R}:=R / \mathrm{m}^{n} R \cong \hat{R} / \mathrm{m}^{n} \hat{R}$, $\bar{g}:=\bar{T} \otimes g, \bar{v}:=\bar{T} \otimes v, \bar{f}: \bar{B} \rightarrow \bar{R}$ the map given by $(g, f)$ and $\bar{w}: \bar{B}^{\prime} \rightarrow \bar{R}$ the map given by $g$ and $\hat{w}$. We have $\bar{w} \bar{v}=\bar{f}$ and so $(\bar{B}, \bar{f})$ has a desingularization with respect to $\bar{g}$. This shows that the hypothesis of $\left[\mathrm{P}_{4}\right]$ Lemma (9.5) hold and so there exist a finite type smooth $T$-algebra $B^{\prime \prime}$ and some $T$-morphisms $\tilde{v}: T \otimes_{k} B \rightarrow B^{\prime \prime}, w^{\prime \prime}: B^{\prime \prime} \rightarrow R$ such that $w^{\prime \prime} \tilde{v}$ is exactly the map given by $g, f$. Since $T$ is in fact a "polynomial" $k$-algebra it follows 
that $w^{\prime \prime}\left(H_{B^{\prime \prime} / k}\right) \not \subset$ 11. Changing $B^{\prime \prime}$ by a suitable localization we can suppose that $B^{\prime \prime}$ is a finite type smooth $k$-algebra. Clearly the composite map $v^{\prime \prime}$, $B \rightarrow T \otimes_{k} B \stackrel{\tilde{v}}{\longrightarrow} B^{\prime \prime}$ satisfies $w^{\prime \prime} v^{\prime \prime}=f$. Using $\left[\mathrm{P}_{4}\right]$ Colollary (3.6) we get a desingularization for $(B, f)$ with respect to $k \subset \rightarrow$. The converse is trivial.

Q.E.D.

\section{\$8. Proof of desingularization theorem}

Let $n$ be a nonnegative integer. We show by induction on $n$ the following four Theorems:

$\left(D F_{n}\right)$ Theorem. Let $(R, m)$ be a formally smooth noetherian local algebra over a field $k . \quad$ Suppose that $\operatorname{dim} R \leqslant n . \quad$ Then $R$ is a filtered inductive limit of standard smooth k-algebras.

$\left(D A_{n}\right)$ Theorem. Let $A \rightarrow A^{\prime}$ be a formally smooth local morphism of noetherian local rings. Suppose that $A$ is artinian and $\operatorname{dim} A^{\prime} \leqslant n$. Then $A^{\prime}$ is a filtered inductive limit of standard smooth A-algebras.

(DT $T_{n}$ ) Theorem. Let $u: A \rightarrow A^{\prime}$ be a morphism of noetherian rings, $B$ a finite type A-algebra, $f: B \rightarrow A^{\prime}$ an A-morphism such that $a:=\sqrt{ } f\left(H_{B / A}\right) A^{\prime}$ $\div A^{\prime}$ and $q$ a minimal prime over-ideal of a of height $\leqslant n$. Suppose that

i) $A_{q}^{\prime}$ is a formally smooth $A_{u-1 q}$-algebra,

ii) for every minimal prime over-ideal $q^{\prime}$ of a the map $A \rightarrow A_{q^{\prime}}^{\prime}$ induced by $u$ is flat and $A_{q^{\prime}}^{\prime} /\left(u^{-1} q^{\prime}\right) A_{q^{\prime}}^{\prime}$ is a regular ring,

iii) $A^{\prime} / q$ is not finite.

Then there exist a finite type A-algebra $B^{\prime}$ and two A-morphisms $v$ : $B \rightarrow B^{\prime}, w: B^{\prime} \rightarrow A^{\prime}$ such that

(*) $w v=f$,

$(* *) \quad \mathfrak{a} \subset \sqrt{ } w\left(H_{B^{\prime} / A}\right) A^{\prime} \not \subset q$.

$\left(D_{n}\right)$ Theorem. Let $u: A \rightarrow A^{\prime}$ be a morphism of noetherian rings, $B$ a finite type A-algebra, $f: B \rightarrow A^{\prime}$ an A-morphism and $\mathfrak{a}_{f}:=\sqrt{ } f\left(H_{B / A}\right) A^{\prime}$. Suppose that for every prime ideal $q \supset \mathfrak{a}_{f}$ from $A^{\prime}, A_{q}^{\prime}$ is a formally smooth $A_{u-1_{q}}$-algebra. Then there exist a finite type A-algebra $B^{\prime}$ and two $A$ morphisms $v: B \rightarrow B^{\prime}, w: B^{\prime} \rightarrow A^{\prime}$ such that

i) $w v=f$,

ii) $a_{f} \subset \sqrt{ } w\left(H_{B^{\prime} / A}\right) A^{\prime}$,

iii) $B_{x^{-1} q}^{\prime}$ is smooth over $A$ for every prime ideal $q \subset A^{\prime}$ satisfying

a) $A^{\prime} / q$ is not finite

b) $\operatorname{ht}(q) \leqslant n$. 
Next we shall prove that for every nonnegative integer $n$ one has

$$
\left(D A_{n}\right) \Longrightarrow\left(D T_{n}\right) \Longrightarrow\left(D_{n}\right) \Longrightarrow\left(D F_{n+1}\right),
$$

the implication $\left(D F_{n}\right) \Rightarrow\left(D A_{n}\right)$ was proved in a particular form in Corollary (5.4); the complete proof is given in Section 9 . Since $\left(D A_{0}\right)$ holds by [P $\mathrm{P}_{4}$ Corollary (3.3) this is enough for our induction. In particular $\left(D T_{n}\right)$ will hold for every $n \in N$ and so the Desingularization Theorem will hold too.

(8.1) Lemma. $\left(D A_{n}\right) \Rightarrow\left(D T_{n}\right)$

Proof. We follow $\left[\mathrm{P}_{4}\right]$ (9.7). Let $\left\{q_{i}\right\}_{1 \leqslant i \leqslant c}, q_{i} \subset A^{\prime}, q_{e}=q$ be the minimal prime over-ideals of $\mathfrak{a}$. Denote $s_{i}:=\mathrm{ht}\left(q_{i} /\left(u^{-1} q_{i}\right) A^{\prime}\right), 1 \leqslant i \leqslant e, s=\sum_{i=1}^{e} s_{i}$, $A^{\prime \prime}:=A^{\prime}[X], X=\left(X_{1}, \cdots, X_{s}\right), q_{i}^{\prime \prime}:=q_{i} A^{\prime \prime}$ and let $h: A^{\prime} \rightarrow A^{\prime \prime}$ be the inclusion. Applying $\left[\mathrm{P}_{4}\right]$ Lemma (9.6), there exist a polynomial $A$-algebra $R$ and an $A$-morphism $g: R \rightarrow A^{\prime \prime}$ such that for every $i, 1 \leqslant i \leqslant e$

1) $g^{-1}(\mathfrak{a}) A_{q_{i}^{\prime \prime}}^{\prime \prime}=q_{i}^{\prime \prime} A_{q_{i}^{\prime \prime}}^{\prime \prime}$,

2) the map $R \rightarrow A_{q_{i}^{\prime}}^{\prime \prime}$ given by $g$ is flat,

3) $k\left(g^{-1} q_{i}^{\prime \prime}\right)=k\left(u^{-1} q_{i}\right)$ (some $X$-es)

By 1) $\left\{q_{i}^{\prime \prime}\right\}_{i}$ are minimal prime over-ideals of $\left(g^{-1} \mathfrak{a}\right) A^{\prime \prime}$. Denote $B^{\prime \prime}:=R \otimes_{4} B$ and let $f^{\prime \prime}: B^{\prime \prime} \rightarrow A^{\prime \prime}$ be the $R$-morphism given by $g$ and $h f$.

Now we want to apply $\left[\mathrm{P}_{4}\right]$ Lemma (9.5) to the case $\left(R, g, A^{\prime \prime}, f^{\prime \prime}, q A^{\prime \prime}\right)$. For this it is necessary to show that

4) for every positive integer $r$ there exist a finite type $\bar{R}_{r}:=R /\left(g^{-1} q\right)^{r}$ algebra $\bar{D}_{r}$ and two $\bar{R}_{r}$-morphisms $\bar{v}_{r}: \bar{B}_{r} \rightarrow \bar{D}_{r}, \bar{B}_{r}:=\bar{R}_{r} \otimes_{R} B^{\prime \prime}, \bar{w}_{r}: \bar{D}_{r} \rightarrow$ $\bar{A}_{r}^{\prime \prime}:=\bar{R}_{r} \otimes_{R} A^{\prime \prime}$ such that $\bar{w}_{r}\left(H_{\bar{D}_{r} / \bar{R}_{r}}\right) \not \subset q \bar{A}_{r}^{\prime \prime}$ and $\bar{w}_{r} \bar{v}_{r}=\bar{B}_{r} \otimes_{B^{\prime \prime}} f^{\prime \prime}$.

Fix $r$. Apply $\left(D A_{n}\right)$ to the case $\tilde{A}:=\left(A /\left(u^{-1} q\right)^{r}\right)_{u-1 q}, \tilde{A}^{\prime}:=\left(A^{\prime} /\left(u^{-1} q\right)^{r} A^{\prime}\right)_{q}$, $\tilde{B}:=\tilde{A} \otimes_{A} B, \tilde{f}: \tilde{B} \rightarrow \tilde{A}^{\prime}$ being given by $\tilde{A} \otimes_{A} f$. Then there exist a finite type smooth $\tilde{A}$-algebra $\tilde{C}$ and two $\tilde{A}$-morphisms $\sigma: \tilde{B} \rightarrow \tilde{C}, \tau: \tilde{C} \rightarrow \tilde{A}^{\prime}$, such that $\tau \sigma=\tilde{f}$. Note that $\bar{C}:=\bar{R}_{r} \otimes_{A} \tilde{C}$ is a finite type smooth $\bar{R}_{r}$-algebra and $\bar{R}_{r} \otimes_{A} \tilde{B}$ is a localization of $\bar{B}_{r}$. We can choose a subring $\bar{D}_{r} \subset \bar{C}$ such that

a) the morphism $\mu: \bar{C} \rightarrow\left(\bar{A}_{r}^{\prime \prime}\right)_{q . A^{\prime \prime}}$ given by $\bar{R}_{r} \otimes g$ and $\tau$, maps $\bar{D}_{r}$ in $\bar{A}_{\prime}^{\prime \prime}$,

b) $\bar{C}$ is a localization of $\bar{D}_{r}$,

c) $\bar{D}_{r} \supset\left(\bar{R}_{r} \otimes_{\Lambda} \sigma\right) \bar{B}_{r}$, the inclusion being of finite type.

Clearly the maps $\bar{v}_{r}: \bar{B}_{r} \rightarrow \bar{D}_{r}, \bar{w}_{r}: \bar{D}_{\imath} \rightarrow \bar{A}_{r}^{\prime \prime}$ given by $\bar{R}_{r} \otimes_{A} \sigma$ and $\mu$, are subject to 4).

By $\left[\mathrm{P}_{4}\right]$ Lemma (9.5) there exist a finite type $A$-algebra $B^{\prime}$ and two $A$ morphisms $v: B \rightarrow B^{\prime}, w^{\prime}: B^{\prime} \rightarrow A^{\prime \prime}$ such that 
5) $w^{\prime} v=h f$

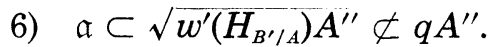

Since $A^{\prime} / q$ is not finite, like in $\left[\mathrm{P}_{4}\right]$ (9.7) there exist some elements $x=$ $\left(x_{1}, \cdots, x_{s}\right) \in A^{\prime s}$ such that the $A^{\prime}$-morphism $\rho_{x}: A^{\prime \prime} \rightarrow A^{\prime}, X \rightarrow x$ satisfies

$$
\rho_{x} w^{\prime}\left(H_{B^{\prime} / A}\right) \not \subset q \text {. }
$$

Take $w=\rho_{x} w^{\prime}$

Q.E.D.

(8.2) Lemma. $\left(D T_{n}\right) \Rightarrow\left(D_{n}\right)$

Proof. We follow the proof of $\left[\mathrm{P}_{4}\right]$ Theorem (5.2). Let $G$ be the set of all prime ideals $q$ from $A^{\prime}$ such that $A^{\prime} / q$ is not finite and $h t(q) \leqslant n$. Consider the Zariski topology on $G$ and apply noetherian induction on $F_{f}:=V\left(a_{f}\right) \cap G$. If $F_{f}=\emptyset$ we have nothing to prove. By induction hypothesis it is enough to show that given $(B, f)$ and a minimal prime over-ideal $q \in F_{f}$ of $\mathfrak{a}_{f}$ there exist a finite type $A$-algebra $B_{1}$ and two $A$ morphisms $h: B \rightarrow B_{1}, f_{1}: B_{1} \rightarrow A^{\prime}$ such that

1) $f_{1} h=f$,

2) $\mathfrak{a}_{f} \subset \mathfrak{a}_{f_{1}} \not \subset q$ and so $V\left(\mathfrak{a}_{f_{1}}\right) \subsetneq V\left(\mathfrak{a}_{f}\right)$.

But this follows from $\left(D T_{n}\right)$.

Q.E.D.

(8.2.1) Remark. $\left(D T_{n}\right)$ plays here the role of $\left[\mathrm{P}_{4}\right]$ Desingularization Lemma.

(8.3) Lemma. $\left(D_{n}\right) \Rightarrow\left(D F_{n+1}\right)$

Proof. Using Lemma (2.2) it is enough to show that given a finite type $k$-algebra $B$ and a $k$-morphism $f: B \rightarrow R$ there exists a desingularization of $(B, f)$. By $\left(D_{n}\right)$ there exist a finite type $k$-algebra $\tilde{B}$ and two $k$-morphisms $v: B \rightarrow \tilde{B}, w: \tilde{B} \rightarrow R$ such that

1) $w v=f$

2) $\tilde{B}_{w-1 q}$ is smooth $k$-algebra for every prime ideal $q \subset R, q \neq \mathfrak{m}$ $\left(A^{\prime} / q\right.$ is not finite because it is not field), i.e. $\sqrt{w\left(H_{\tilde{B} / k}\right) R}=\mathfrak{m}$.

If $K:=R / \mathfrak{m}$ is a separable field extension of $k$ then $(\tilde{B}, w)$ has a desingularization by $\left[\mathrm{P}_{4}\right]$ Theorem $(5.2)$ and so $(B, f)$ has too. Otherwise it is enough to apply Theorem (7.3).

Q.E.D.

\section{§9. Countable rings and strong algebraically pure morphisms}

(9.1) A ring morphism $A \rightarrow B$ is called strong algebraically pure if for every finite system of polynomials $f$ from $A[X], X=\left(X_{1}, \cdots, X_{m}\right)$ and 
for every finite set of finite systems of polynomials $\left\{g_{i}\right\}_{1 \leqslant i \leqslant r}$ from $A[X, Y]$, $Y=\left(Y_{1}, \cdots, Y_{N}\right)$ the following conditions are equivalent:

i) $f$ has a solution $x$ in $A$ such that for every $i, 1 \leqslant i \leqslant r$ the system $g_{i}(x, Y)$ has no solution in $A$,

ii) $f$ has a solution $x$ in $B$ such that for every $i, 1 \leqslant i \leqslant r$ the system $g_{i}(x, Y)$ has no solution in $B$.

Clearly a strong algebraically pure morphism is algebraically pure. In [BNP] such morphisms are called $T^{\prime \prime}$-existentially complete, properties and examples can be found there. Let $A$ be a noetherian local ring and $\hat{A}$ its completion. The canonical map $A \rightarrow \hat{A}$ is strong algebraically pure iff it is algebraically pure (i.e. iff $A$ is an AP-ring).

(9.1) Lemma. Let $u: A \rightarrow B$ be a strong algebraically pure morphism. Suppose that $B$ is a noetherian local ring. Then it holds

i) $A$ is a noetherian local ring, its maximal ideal generates the maximal ideal of $B$ and $\operatorname{dim} A=\operatorname{dim} B$,

ii) $u$ is formally smooth,

iii) every prime ideal $q \subset A$ extends to a prime ideal $q B$ and the field extension $k(q) \rightarrow k(q B)$ is strong algebraically pure, in particular separable.

Proof. First we shall see that for every ideal $\mathfrak{a} \subset A$ we have $\mathfrak{a}=$ $u^{-1}(\mathfrak{a} B)$. Let $y \in u^{-1}(\mathfrak{a} B)$. Then $u(y)=\sum_{i=1}^{s} d_{i} x_{i}^{\prime}$ for some $d_{i} \in \mathfrak{a}, x_{i}^{\prime} \in B$. Since $u$ is algebraically pure there exists $x=\left(x_{1}, \cdots, x_{s}\right) \in A^{s}$ such that $y=\sum_{i=1}^{s} d_{i} x_{i}$. Thus $y \in \mathfrak{a}$.

Let $I \subset A$ be an arbitrary ideal. Then there exists a finitely generated subideal $J \subset I$ such that $I B=J B, B$ being noetherian. We have $I=u^{-1}(I B)$ $=u^{-1}(J B)=J$ and so $I$ is finitely generated. Thus $A$ is a noetherian ring.

Now, applying [BNP] Corollary (4.6.2), we get $u$ faithfully flat and by Proposition (4.6.8) [ibid], $A$ is local and its maximal ideal generates the maximal ideal of $B$. Also by Proposition (4.6.1) [ibid] we have iii) and by [EGA] part 1 (19.7.1), the map $A \rightarrow B$ is formally smooth.

Q.E.D.

The above Lemma and some other results from [BNP] give a hope for a positive answer to the following

(9.2) Question. Is a strong algebraically pure morphism of noetherian rings a regular morphism?

The following Lemma is close to Löwenheim-Skolem Theorem-an well known result from Model Theory. 
(9.3) Lemma. Let $A$ be a ring and $S \subset A$ a countable subset. Then there exists a countable subring $C$ of $A$ containing $S$ such that the inclusion $C \rightleftharpoons A$ is strong algebraically pure.

Proof. We shall construct by induction a family of countable subrings $\left(C_{j}\right)_{\jmath \in N}$ of $A$ containing $S$ and such that for every finite systems of polynomials $f(X), g_{i}(X, Y)_{1 \leqslant i \leqslant r}$ in $X=\left(X_{1}, \cdots, X_{n}\right), Y=\left(Y_{1}, \cdots, Y_{N}\right) ; r, n, N \in N$ over $C_{j}$ such that $f$ has a solution $x$ in $A$ which makes every system of equations $g_{i}(x, Y)=0,1 \leqslant i \leqslant r$ incompatible in $A$, there exists a solution $x^{\prime}$ of $f$ in $C_{j+1}$ which makes every system $g_{i}\left(x^{\prime}, Y\right)=0$ incompatible in $A$. Let $C_{1}$ be the subring generated by $S$ in $A$. Given $C_{j}, j \geqslant 1$ consider the countable family of all possible finite systems of polynomials $f,\left\{g_{i}\right\}_{1 \leqslant i \leqslant r}$, $r \in N$ such that there exist in $A$ a solution $x_{f, g}$ of $f$ which makes

$$
g_{i}\left(x_{f, g}, Y\right)=0
$$

incompatible in $A$ for every $i, 1 \leqslant i \leqslant r$. Put $C_{\jmath+1}=C_{\jmath}\left[\left\{x_{f, g}\right\}_{f, g}\right]$. Clearly $C:=\bigcup_{i \in N} C_{j}$ satisfies our Lemma.

Q.E.D.

The following result is necessary in Section 8; since its proof use some facts from "countable rings" we present it here.

(9.4) Proposition. Let $n$ be a positive integer. The following conditions are equivalent

i) Every formally smooth, n-dimensional, noetherian local algebra over a field $k$ is a filtered inductive limit of standard smooth $k$-algebras

ii) Every formally smooth, n-dimensional, noetherian local algebra over an artinian local ring $A$ is a filtered inductive limit of standard smooth A-algebras.

Proof. Clearly ii) $\Rightarrow$ i). First we show i) $\Rightarrow$ ii) when $A$ is a countable ring. Let $A^{\prime}$ be a formally smooth, $n$-dimensional noetherian local $A$ algebra. By Lemma (9.3) we can express $A^{\prime}$ as a filtered inductive union of countable sub- $A$-algebras $C$ of $A^{\prime}$ such that the inclusion $C \subset A^{\prime}$ is strong algebraically pure, let us say $A^{\prime}=\cup_{i \in I} C_{i}$. Then the inclusions $C_{i} \longrightarrow A^{\prime}$ are faithfully flat by Lemma (9.1) and so $C_{i}$ are formally smooth $A$-algebras (use [M] (21.D) like in [M] (33.B)).

Let $\mathfrak{m}$ be the maximal ideal from $A$. Using i) we can express $C_{i} / \mathfrak{m} C_{i}$ as a filtered inductive limit of standard smooth $k:=A / \mathrm{m}$-algebras. Using Corollary (5.4) we can express also every $C_{i}, i \in I$ as a filtered inductive limit of standard smooth $A$-algebras and so we get ii). 
Now suppose that $A$ is not countable. Let $B$ be a finite type $A$ algebra and $f: B \rightarrow A^{\prime}$ an $A$-morphism. By Lemma (2.2) it is enough to show that $(B, f)$ has a desingularization. As above using Lemmas (9.1) and (9.3) we can express $A$ as a filtered inductive union of countable subrings, let us say $A=\bigcup_{i \in I} D_{i}$, such that the inclusions $D_{i} \rightarrow A, i \in I$ are formally smooth. Then there exists a finite type $D_{i}$-algebra $E_{\imath}$ for a certain $i \in I$ such that $A \otimes_{D_{i}} E_{i} \cong B$. Let $f_{\imath}: E_{i} \rightarrow A^{\prime}$ be the composite map $E_{\imath} \rightarrow$ $A \otimes_{D_{i}} E_{i} \cong B \stackrel{f}{\longrightarrow} A^{\prime}$. Since $D_{i}$ is countable there exist a standard smooth $D_{i}$-algebra $E_{i}^{\prime}$ and two $D_{i}$-morphisms $v_{i}: E_{i} \rightarrow E_{i}^{\prime}, w_{i}: E_{i}^{\prime} \rightarrow A^{\prime}$ such that $w_{i} v_{i}=f_{i}$. Take $B^{\prime}:=B \otimes_{E_{i}} E_{i}^{\prime}, v:=B \otimes_{E_{i}} v_{i}$ and let $w: B^{\prime} \rightarrow A^{\prime}$ be the map given by $f$ and $w_{i}$. Clearly $\left(v, B^{\prime}, w\right)$ form a desingularization for $(B, f)$.

Q.E.D.

\section{$\S 10$. Some remarks on scalar extension left adjoint}

Let $R=R^{\prime}$ be an extension of rings such that $R^{\prime}$ is a finitely generated free $R$-module. Then a well known result (see e.g. [G], or [Gr]) says the following

(10.1) Lemma. There exists a functor $\mathscr{F}_{R^{\prime} / R}$ from the category of $R^{\prime}$ algebras of finite type to the category of $R$-algebras of finite type which is a left adjoint to the extension of scalars from $R$ to $R^{\prime}$.

Proof (Sketch). Let $e_{1}, \cdots, e_{d}$ be a base of $R^{\prime}$ over $R$ and $B:=R^{\prime}[Y] /(f)$, $Y=\left(Y_{1}, \cdots, Y_{n}\right), f=\left(f_{i}\right)_{i \in I}$. Substituting in $f_{i}$ the variables $\left(Y_{k}\right)$ by $\left(Y_{k}^{+}\right)$, $Y_{k}^{+}:=\sum_{j=1}^{d} Z_{k j} e_{j}, Z=\left(Z_{k j}\right)$ being some new variables we can express $f_{i}\left(Y_{i k}^{+}\right)$ in the following form

$$
f_{i}\left(Y_{k}^{+}\right)=\sum_{s=1}^{l} g_{i s}(Z) e_{s},
$$

where $\left(g_{i s}\right)$ are polynomials in $Z$ over $R$. Take $\mathscr{F}(B)=R[Z] /(g), g=\left(g_{i s}\right)_{i, s}$.

Now, let $C$ be an arbitrary $R$-algebra and $u: B \rightarrow C \otimes_{R} R^{\prime}$ a $R^{\prime}$-morphism. The map $u$ is completely defined by a solution $y=\left(y_{1}, \cdots, y_{n}\right)$ of the system $f$ in $C \otimes_{R} R^{\prime}$, let us say $y_{i}=\sum_{t=1}^{d} z_{\imath t} \otimes e_{t}, z_{i t} \in C$. Clearly $y$ is a solution of $f$ in $C \otimes_{R} R^{\prime}$ iff $z=\left(z_{i t}\right)$ is a solution of $g$ in $C$. The $R$ morphism $v: \mathscr{F}(B) \rightarrow C, Z \rightarrow z$ is functorially associated to $u$. Q.E.D.

(10.2) Lemma. Let $C$ be a finite type $R$-algebra and $C^{\prime}:=R^{\prime} \otimes_{R} C$. Then there exists the following functorial isomorphism

$$
\mathscr{F}_{C^{\prime} / C}(-) \cong C \otimes_{\tilde{\mathcal{F}}_{R^{\prime} / R^{\left(C^{\prime}\right)}}}\left(\mathscr{F}_{R^{\prime} / R} \alpha_{*}\right)(-)
$$


where $\alpha_{*}$ is the scalar restriction from $C^{\prime}$ to $R^{\prime}$ and the structure of $\mathscr{F}_{R^{\prime} / R}\left(C^{\prime}\right)$ algebra on $C$ is given by the adjunction map $\varphi: \mathscr{F}_{R^{\prime} / R}\left(C^{\prime}\right) \rightarrow C$.

Proof. It is enough to show that the functor $G:=C \otimes_{\mathscr{F}_{R^{\prime} / R}\left(C^{\prime}\right)}$ $\left(\mathscr{F}_{R^{\prime} / R} \alpha_{*}\right)(-)$ is a left adjoint to the extension of scalars from $C$ to $C^{\prime}$. Let $D^{\prime}$ be a finite type $C^{\prime}$-algebra, $v^{\prime}: C^{\prime} \rightarrow D^{\prime}$ its structural morphism, $E$ an $R$-algebra, $E^{\prime}:=R^{\prime} \otimes_{R} E, u: C \rightarrow E$ a $R$-morphism, $u^{\prime}:=R^{\prime} \otimes_{R} u$ and $w^{\prime}: D^{\prime} \rightarrow E^{\prime}$ a $C^{\prime}$-morphism, i.e. a $R^{\prime}$-morphism such that $w^{\prime} v^{\prime}=u^{\prime}$. The $R$-morphism $\tilde{w}:\left(\mathscr{F}_{R^{\prime} / R} \alpha_{*}\right)\left(D^{\prime}\right) \rightarrow E$ corresponding to $w^{\prime}$ by adjunction satisfies

$$
\tilde{w}\left(\mathscr{F}_{R^{\prime} / R} \alpha_{*}\right) v^{\prime}=u \varphi
$$

and so there exists an unique $C$-morphism $w: G\left(D^{\prime}\right) \rightarrow E$ extending $\tilde{w}$. The correspondence $w^{\prime} \rightarrow w$ is bijective and functorial.

Q.E.D.

(10.3) Proposition. Let $u ; A \rightarrow A^{\prime}$ be a ring morphism, $B$ a finite type A-algebra, $f: B \rightarrow A^{\prime}$ an A-morphism, $\tilde{A}$ a finite free A-algebra, $\tilde{B}:=\tilde{A} \otimes_{A} B$, $\tilde{f}:=\tilde{A} \otimes_{A} f$ and $\tilde{A}^{\prime}:=\tilde{A} \otimes_{A} A^{\prime}$. Suppose that there exist a finite type smooth $\tilde{A}$-algebra $\tilde{C}$ and two $\tilde{A}$-morphisms $\tilde{v}: \tilde{B} \rightarrow \tilde{C}, \tilde{w}: \tilde{C} \rightarrow \tilde{A}^{\prime}$ such that $\tilde{w} \tilde{v}=\tilde{f}$. Then there exist a finite type smooth A-algebra $C$ and two A-morphism $v$ : $B \rightarrow C, w: C \rightarrow A^{\prime}$ such that $w v=f$.

Proof. Take $C:=\mathscr{F}_{\tilde{B} / B}(\tilde{C})$ and let $v: B \rightarrow C$ be the algebra structure morphism. By "adjunction" there exists a $B$-morphism $w: C \rightarrow A$ ' corresponding to $\tilde{w}$ (this holds though $A^{\prime}$ is not a finite type $B$-algebra cf. the proof of Lemma (10.1)), the algebra structure of $A^{\prime}$ over $B$ being given by $f$. Thus $w v=f$.

On the other hand, we note that $\mathscr{F}_{\tilde{A} / A}(\tilde{C})$ is a finite type smooth $A$ algebra. Indeed, for every surjective morphism of $A$-algebras $h: K \rightarrow M$ such that $\operatorname{Ker} h$ is nilpotent we have following commutative diagram

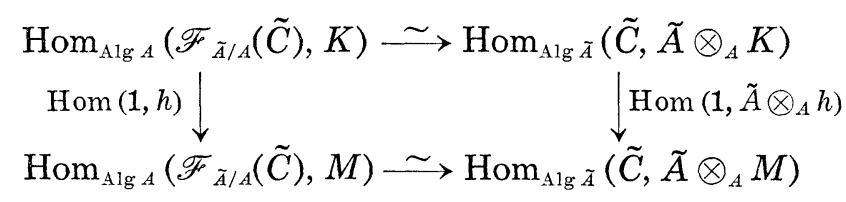

where the bases are isomorphisms and the second vertical map is surjective by smoothness. Then the first vertical map is also surjective and so $\mathscr{F}_{\tilde{A} / A}(\tilde{C})$ is smooth over $A$.

Now using Lemma (10.2) the following square is cocartesian 


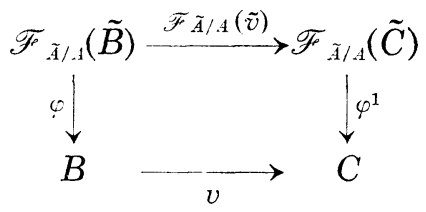

$\varphi$ being an adjunction morphism. By adjunction $\tilde{A} \otimes_{.1} \varphi$ has a section, namely the adjunction morphism $v_{\tilde{B}}: \tilde{B} \rightarrow \tilde{A} \otimes_{A} \mathscr{F}_{\tilde{A} / 1}(\tilde{B})$. Then $\tilde{A} \otimes_{A} \varphi^{\prime}$ has also a section and so $\tilde{A} \otimes_{A} C$ is a direct factor of $\tilde{A} \otimes_{-1} \tilde{F}_{\tilde{I} / L}(\tilde{C})$. Thus $\tilde{A} \otimes_{A} C$ is smooth over $\tilde{A}$. Using descent property of smoothness [EGA] Part 4, (17.7.1) ( $\tilde{A}$ is faithfully flat over $A$ ) we get $C$ smooth over $A$.

Q.E.D.

(10.4) Proof of Lemma (7.4). Using the above Lemma we can find a finite type smooth $A$-algebra $B^{\prime}$ and two $A$-morphisms $v: B \rightarrow B^{\prime}, w: B^{\prime} \rightarrow$ $A^{\prime}$ such that $w v=f$. If $B^{\prime}$ is not standard smooth, apply $\left[\mathrm{P}_{4}\right]$ Corollary (3.6).

Q.E.D.

Added in proof. The technique from Section 7 was improved by V. Nica and the author in "A structure theorem on formally smooth morphisms in positive characteristic" (J. of Algebra vol. 100, $\mathrm{n}^{\circ} 2$ (1986), 436-455) for to get an easier proof to our Theorem (2.3).

Added in Proof. I am indebted to J. Nishimura for pointing out that the proof of $\left[\mathrm{P}_{4}\right]$ Theorem (5.2), when $F$ contains prime ideals whose residue fields are finite, was wrong. That proof is to be replaced by the following one. Applying Desingularization Lemma several times we reduce to the case when $a_{f}=\bigcap_{i=1}^{e} q_{i}$ for some $q_{i} \in F$ such that $\left(A^{\prime} q_{i}\right)_{i}$ are finite fields. Let $j: A^{\prime} \rightarrow A^{\prime}[X]$ be the canonical map. Applying the quoted Lemma several times we find an $A$-algebra $B^{\prime \prime}$ of finite type and two $A$ morphisms $v^{\prime \prime}: B \rightarrow B^{\prime \prime}, w^{\prime \prime}: B^{\prime \prime} \rightarrow A^{\prime}[X]$ such that $w^{\prime \prime} v^{\prime \prime}=$ if and

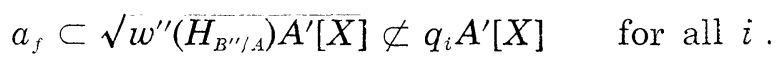

Then there exists a polynomial $P \in w^{\prime \prime}\left(H_{B^{\prime \prime} / 4}\right)$ which is not in $\bigcup_{i=1}^{e} q_{i} A^{\prime}[X]$. By Chinese Remainder Theorem we find a monic polynomial $G$ in $A^{\prime}[T]$ of degree $>\operatorname{deg} P$ which is irreducible in $A^{\prime} / q_{i}[T]$ for all $i$. Put $\tilde{A}=A[T] /(G)$, $\tilde{B}=\tilde{A} \otimes B, \tilde{B}^{\prime \prime}=\tilde{A} \otimes B^{\prime \prime}, \tilde{A}^{\prime}=\tilde{A} \otimes A^{\prime}, \tilde{f}=\tilde{A} \otimes f, \tilde{w}^{\prime \prime}=\tilde{A} \otimes w^{\prime \prime}$. Since $\left(A^{\prime} / q_{i}\right)_{i}$ are fields of cardinal $>\operatorname{deg} P$ there exists $x \in \tilde{A}^{\prime}$, such that $P(x)$ $\notin \bigcup_{i=1}^{e} q_{i}$. We have $\tilde{w}\left(H_{\tilde{B}^{\prime \prime} / \tilde{A}}\right)=\tilde{A}^{\prime}, \tilde{w}: \tilde{B}^{\prime \prime} \rightarrow \tilde{A}^{\prime}$ being given by $b \leadsto$ $\tilde{w}^{\prime \prime}(b)(x)$. Thus $\left(\tilde{B}^{\prime \prime}, \tilde{w}\right)$, and so $(\tilde{B}, \tilde{f})$, has a desingularization with respect 
to $\tilde{A} \otimes u$ by $\left[P_{t}\right]$ Corollary (3.6). Then $(B, f)$ has a desingularization by our Lemma (7.4).

\section{REFERENCES}

[An ${ }_{1}$ André, M., Homologie des algèbres commutatives, Springer-Verlag, Berlin 1974.

[An ${ }_{2}$ ] - Localisation de la lissité formelle, Manuscripta Math., 13 (1974), $297-$ 307 .

[An $\left.n_{3}\right]$ - Artin's theorem on the solution of analytic equations in positive characteristic, Manuscripta Math., 15, (1975), 314-348.

[A $A_{1}$ Artin, M., On the solution of analytic equations, Invent. math., 5 (1968), $277-291$

[A $\left.\mathrm{A}_{2}\right]$ - Algebraic approximation of structures over complete local rings, Publ. Math. IHES, 36 (1969), 23-58.

$\left[\mathrm{A}_{3}\right] \quad-$ Construction techniques for algebraic spaces, Actes Congrès Intern. Math., t. 1 (1970), 419-423.

[AD] Artin, M. and Denef, J., Smoothing of a ring homomorphism along a section, Arithmetic and Geometry, vol. II, Birkhäuser, Boston (1983), 5-32.

[BNP] Basarab, S., Nica, V. and Popescu, D., Approximation properties and existential completeness for ring morphisms, Manuscripta Math., 33 (1981), 227-282.

[BDLV] Becker, J., Denef, J., Lipshitz, L. and van den Dries, L., Ultraproducts and approximation in local rings I, Invent. Math., 51 (1979), 189-203.

[BDL] Becker, J., Denef, J. and Lipshitz, L., The approximation property for some 5-dimensional henselian rings, Trans. Amer. Math. Soc., 276, no. 1 (1983), 301-309.

[BR] Brezuleanu, A. and Radu, N., Sur la localisation de la lissité formelle, C.R. Acad. Sci. Paris, Sér. A, t. 276 (1973), 439-441.

[B] Brown, M. L., Artin's approximation property, Thesis, Cambridge Peterhouse, August 1979.

[CP] Cipu, M. and Popescu, D., Some extensions of Néron's $p$-desingularization and approximation, Rev. Roumaine Math. Pures Appl., t. XXIV, no. 10 (1981), 1299-1304.

[DL] Denef, J. and Lipshitz, L., Ultraproducts and approximation in local rings II, Math. Ann., 253 (1980), 1-28.

[E] Elkik, R., Solutions d'equations á coefficients dans un anneaux hensélien, Ann. Sci. École Norm. Sup. $4^{\circ}$ serie, t. 6 (1973), 533-604.

[Ga] Gabrielov, A. M., The formal relations between analytic functions (Russian), Funktional Anal. i Priložen., 5 (1971), no. 4, 64-65.

[Gr] Greenberg, M., Rational points in henselian discrete valuation rings, Publ. Math. IHES, 31 (1966), 59-64.

[EGA] Grothendieck, A. and Dieudonné, J., Éléments de géometrie algébrique, IV, Parts 1 and 4, Publ. Math. IHES, 1964.

[G] Grothendieck, A., Technique de descente II, Sém. Bourbaki, 195 (1959-1960).

[J] Jähner, U., Der Satz von M. Artin über die Lösungen analytischer Gleichungen mit Koeffizienten in einem Körper beliebiger Charakteristik, Arch. Math., XXIX (1977), 485-490.

[KPR] Kurke, H., Pfister, G., and Roczen, M., Henselsche Ringe and algebraische Geometrie, VEB, Berlin, 1975. 
[KMPPR] Kurke, H., Mostowski, T., Pfister, G., Popescu, D., and Roczen, M., Die Approximationseigenschaft lokaler Ringe, Lect. Notes in Math., 634, SpringerVerlag, Berlin, 1978.

[M] Matsumura, H., Commutative algebra, Benjamin, New York, 1980.

[PP $\left.{ }_{1}\right] \quad$ Pfister, G. and Popescu, D., Die strenge Approximationseigenschaft lokaler Ringe, Invent. Math., 30 (1975), 145-174.

$\left[\mathrm{PP}_{2}\right] \quad-$, On three dimensional local rings with the property of approximation, Rev. Roumaine Math. Pures Appl. t. XXVI, no. 2 (1981), 301-307.

[ $\left.\mathrm{P}_{1}\right] \quad$ Popescu, D., Algebraically pure morphisms, Rev. Roumaine Math. Pures Appl., t. XXIV, no. 6 (1979), 947-977.

$\left[\mathrm{P}_{2}\right] \quad-$ A remark on two dimensional local rings with the property of approximation, Math. Z., 173 (1980), 235-240.

$\left[\mathrm{P}_{3}\right] \quad$ - Global form of Néron's $p$-desingularization and approximation, Proceedings "Week of Algebraic Geometry" Bucharest, June 30-July 6, 1980, Teubner Texte Band 40, Leipzig, 1981.

$\left[\mathrm{P}_{4}\right] \quad-$ General Néron desingularization, Nagoya Math. J., 100 (1985), 97-126.

$\left[\mathrm{P}_{5}\right] \quad-$ On Zariski's Uniformization Theorem, Algebraic Geometry, Bucharest 1982, Proceedings, Lect. Notes in Math., 1056, Springer-Verlag, Berlin, 1984.

[R] Radu, N., Sur une critère de lissité formelle, Bull. Math. Soc. Sci. Math. R.S. Roumaine t. 21, 68, no. 1-2 (1977), 133-135.

[Ra] Raynaud, M., Anneaux locaux henséliens, Lect. Notes in Math., 169, SpringerVerlag, Berlin, 1970.

[Ro] Rotthaus, C., Potenzreihenerweiterung und formale Fasern in lokalen Ringen mit Approximationseigenschaft., Manuscripta Math., 42 (1983), 53-65.

[S] Schemmel, K. P., Eine notwendige und hinreichende Bedingung für die Approximationseigenschaft analytischer Potenzreihenringe über einem Körper beliebiger characteristik, Rev. Roumaine Math. Pures Appl., t. XXVII, no. 8 (1982), 875-884.

[ZS] Zariski, O. and Samuel, P., Commutative Algebra II, D. van Nostrand Company Inc., New York 1960.

\section{INCREST}

Department of Mathematics

Bd. Păcii 220, 79622 Bucharest

ROMANIA 